\title{
Die Stellung der Religion in den soziokulturellen Strukturen
}

\author{
Von HARALDS BIEZAIS
}

1. Wenn man die speziellen Probleme in den Beiträgen dieses Symposiums erörtern will, die mit dem Hauptthema: die dynamischen Prozesse im religiösen Leben und deren Gegensatz - die Bildung von Institutionen - verbunden sind, muß man einige grundsätzliche Tatsachen im Auge behalten. Daher möchte ich vor allem auf drei solcher Zusammenhänge aufmerksam machen.

1.1. Zu den grundlegenden Tatsachen, die man nicht übersehen darf, gehört die Erkenntnis, daß die Religion einen integrierenden Bestandteil der soziokulturellen Struktur ihrer Zeit bildet ${ }^{1}$. Diese Feststellung ist weder neu noch revolutionierend. Doch ist daran zu erinnern, weil sie zu oft vergessen wird, während man sich auf andere Zusammenhänge und Motivierungen beruft. Das geschieht aus Interessen heraus, die in keinem direkten Zusammenhang mit der Forschung stehen. In unserem Fall sind nur empirisch feststellbare Erscheinungen in Betracht zu ziehen. Diese Bedingung ist nicht nur von der Religionswissenschaft sozusagen um ihrer selbst willen zu stellen, dazu zwingt auch die interdisziplinäre Zusammenarbeit mit anderen Forschungsrichtungen, vor allem der Psychologie, Soziologie, Sozialanthropologie u.a. Eine Entfernung von der empirischen Grundlage würde bedeuten, sich außerhalb des wissenschaftstheoretischen Rahmens zu stellen, innerhalb dessen die erwähnten Wissenschaften tätig sind. Ich sagte eben, daß diese Forderung weder neu noch revolutionär sei. Das war sie aber zu Anfang dieses Jahrhunderts, als sie von Söderblom geäußert wurde ${ }^{2}$.

\footnotetext{
${ }^{1}$ Bereits 1936 wies Ogburn in seinem Aufsatz ,Stationary and Changing Societies" (AJS 42) auf den engen Zusammenhang zwischen den sozialen und den religiösen Wandlungen hin. Cf. Ogburn $99 \mathrm{ff}$., auch Steinbacher 82; Mensching 17, $20 \mathrm{f}$.

${ }^{2}$ Söderblom $6 \mathrm{f} .:$,,Så långt mänsklig forskning strängt taget kan nå, har religionen sin källa i människosjälen. Om vad som ligger där bakom kunna olika meningar råda." Wie schwer es Söderblom selbst hatte, in seinen Forschungen diese Forderung aufrechtzuerhalten, zeigen die Schwäche seiner erkenntnistheoretischen Position und die Widersprüche, auf die bereits mehr-
} 
Doch ist die Tendenz, außerempirische Faktoren zur Erklärung religiöser Erscheinungen heranzuziehen, nicht geschwunden, sondern hat sich zuweilen sogar noch verstärkt. Früher war das Interesse für fremde Religionen von der Praxis her bestimmt. Es galt, Informationen über die fremden Religionen für Missionierungszwecke zu erhalten ${ }^{3}$. Jetzt ist die Tendenz zu beobachten, die Religionsforschung verschiedenen ideologischen Forderungen zu unterwerfen, sei es, daß sie in der Ideologie des kommunistischen Marxismus ${ }^{4}$ oder in der dogmatischen Theologie begründet sind. Letztere werden besonders in den wiederholt veröffentlichten Studien "Theologie der Religionsgeschichte" deutlich. Solche Bestrebungen erschweren eine objektive Religionsforschung.

1.2. Der zweite Umstand, den es zu berücksichtigen gilt, betrifft den dynamischen Charakter des menschlichen Wesens. Es klingt fast banal, wenn wir hier daran erinnern, daß der Mensch ein dynamisches Wesen ist. Er ist wie kein anderes Wesen in der Lage, sein Dasein mit kritischem Bewußtsein zu erleben, sich dazu zu bekennen und es zu überprüfen. Dadurch bestätigt sich ihm um so mehr sein dynamischer Charakter. Wenn man erkannt hat, daß der Mensch seinem Wesen nach eine dynamische Erscheinung ist, braucht man das nicht mehr besonders zu betonen. Hier muß die Aufmerksamkeit auf das Verständnis der durch diese Dynamik ausgelösten Erscheinungen gerichtet werden. Es besteht ein grosser Unter-

fach hingewiesen wurde. Cf. Holmström 176 s., 419f., 422; auch Biezais 1971, 7 und id. 1971 a, $37 \mathrm{f}$. Aber daß diese Position richtig ist, hat gerade in letzter Zeit Körber, 307, besonders unterstrichen.

${ }^{3}$ Hier können die Äußerungen von Vicedom $12 \mathrm{f}$., erwähnt werden; Rosenkranz 301: ,,Christlicher Glaube angesichts der Weltreligionen, d. h. der Glaube an den persönlichen, lebendigen Gott, der in der Geschichte, in Jesus von Nazareth, Mensch geworden und den Menschen in ihrem Erdendasein nahe ist, ist Glaubenserfahrung, seine Wirkung ist Daseinsverwandlung. Dies mitzuteilen, in existentieller Konfrontation zu bezeugen und damit den östlichen Menschen und den westlichen Menschen zur Entscheidung für den Vater Jesu Christi zu rufen, ist seine Bestimmung. In eine Menschheit hinein, auf deren Herzen die Decke der Selbsterlösung liegt, tritt er mit 'unverhülltem Antlitz', auf dem sich der Widerschein der 'Herrlichkeit Gottes' spiegelt (2. Kor. 3,15-18), der Glanz der 'Güte und Leutseligkeit Gottes, unseres Heilandes' (Tit. 3,4), der Triumph über die finsteren Mächte. Der christliche Glaube ist der Sieg, der die Welt überwunden hat (i. Joh. 5,4), der Triumph über die finsteren Mächte des menschlichen Daseins, die Erhöhung in die königliche Freiheit des Menschen von sich selbst, die aus dem lebt, mit dem er steht und fält: Jesus Christus. Das muß er seinem Gesprächspartner sagen; denn das ist sein Dienst der Versöhnung.“ Ähnlich auch Holsten $207 \mathrm{f}$.

${ }^{4}$ Über diese Erscheinung ist sehr viel geschrieben worden; cf. auch Biezais $1965 \mathrm{a}, 123 \mathrm{ff}$. 
schied zwischen den religiösen Vorstellungen der altägyptischen im Neuem Reich, sowie deren Niederschlag in den kultischen Dokumenten jener Zeit, und den religiösen Anschauungen ihrer chinesischen Zeitgenossen der Hiang-Dynastie. Wenn man sich von jenen fernen Zeiten in die Gegenwart versetzt, kann man die offensichtlichen Unterschiede in den religiösen Vorstellungen und im kultischen Leben erkennen, wie sie sich beispielsweise hier im Dom zu Åbo und in dem auf den Neuen Hebriden bekannten Kult Nagriamel abspielen. In beiden Fällen hat man es mit einem christlichen Erbe zu tun. Ferner handelt es sich in beiden Fällen um menschliche Wesen. Weiter vollzieht sich jeweils ein dynamischer Prozeß, der sich innerhalb einer bestimmten kultischen Einheit entwickelt hat. Und dennoch, welche Unterschiede! Das bedeutet, daß die Verschiedenheit andere Ursachen und Zusammenhänge haben muß, die nicht nur durch das Wesen des individuellen Menschen bestimmt sind. Wenn man in diese religiösen Vorstellungen Einblick nimmt, entdeckt man mehrere Besonderheiten. Sie unterscheiden sich nicht nur durch den Inhalt, sondern auch in ihrer formalen Struktur. Ebenso sind auch die zeitlichen Gegebenheiten zu berücksichtigen, denn sowohl die einen wie die anderen Vorstellungen sind historische Erscheinungen, die sich im Laufe der Zeit entwickelt haben. Diese beiden Umstände sind besonders $\mathrm{zu}$ beachten, wenn man vom dynamischen Charakter religiöser Erscheinungen spricht. Dieser Hinweis soll helfen, das anfangs Gesagte über die Einbeziehung religiöser Vorstellungen in den kulturellen Gesamtzusammenhang besser zu verstehen. Hier wird die Entwicklung von Strukturen und Formen einer Religion im Verlauf der Zeit sichtbar.

1.3. Aus dem vorhin Gesagten könnte man auf eine Beschränkung der Religion auf die soziokulturellen Faktoren schließen. Daher ist hier die Frage zu stellen nach der besonderen Funktion und Stellung der Religion im kulturellen Gesamtzusammenhang. Oft spricht man von der Kultur als einer im Verlauf der Zeit und auch in der Bedeutung, d.h. ihrem Wert nach zweitrangig gewordenen Erscheinung. Es ist genug darüber geschrieben worden, was unter Kultur zu verstehen ist. Die Definition des Begriffes Kultur bereitet die gleichen Schwierigkeiten wie die des Begriffes Religion. Für das hier zu besprechende Thema ist das weniger von Bedeutung, daher lassen wir die Diskussion darüber beiseite ${ }^{5}$. Der Zusammenhang von Kultur

${ }^{5}$ Die beste Übersicht über die verschiedenen Standpunkte zur Erklärung des Begriffs Kultur bietet immer noch Kroeber $77 \mathrm{ff}$; cf. auch Tillich $13 \mathrm{ff}$. 
und Religion hat besonders auch das traditionelle theologische Denken beschäftigt. Die Lösung dieses Problems erschien einfach, fast zu einfach. Wir erwähnen hier nur ein Beispiel, die Ansichten des bedeutenden Vertreters der Kulturtheologie Tillich: ,Religion als das, was uns unbedingt angeht, ist die sinngebende Substanz der Kultur, und Kultur ist die Gesamtheit der Formen, in denen das Grundanliegen der Religion seinen Ausdruck findet. Kurz gefaßt: Religion ist die Substanz der Kultur, und Kultur ist die Form der Religion. Eine solche Auffassung verhindert endgültig einen Dualismus von Religion und Kultur. Jeder religiöse Akt, nicht nur in der organisierten Religion, sondern auch im geheimsten Winkel unserer Seele, ist kulturell geformt. ${ }^{66}$ Das Problem ist jedoch nocht so einfach. Mit der Behauptung, daß die Kultur nur ein formaler Ausdruck der Suche des Menschen nach einer letzten Antwort auf die Frage nach dem Sinn des Lebens sei, wird der Kulturbegriff seines Inhalts entleert. Die Äußerung autonomer geistiger Aktivität wird so zu einem sekundären Ausdrucksmittel eines religiösen Vorgangs. Demgegenüber ist es nicht möglich, der Religion, wenn man sie als soziokulturelle Erscheinung betrachtet, in der Bewertung einen besonderen Rang vor allen anderen kulturellen Äußerungen (wie der Kunst im weitesten Sinne, der Wissenschaft usw.) einzuräumen ${ }^{7}$. Das bedeutet nicht, den Charakter sui generis der Religion zu leugnen. Diese ist eine besondere Erscheinung im Gesamtzusammen-

\footnotetext{
${ }^{6}$ Tillich $101 \mathrm{f}$. Diese Ansicht hat Dillenberger, 31, näher präzisiert: ,It is imperative that we not misunderstand what Tillich meant by religion. It is not religion in general, nor the concrete religions of mankind. It refers rather to the dimension of depth or of ultimacy which informs the heart of a culture. Religion is ultimate concern; that is, it is that which is the ultimate meaning in the culture [...]. Tillich carefully distinguished preliminary concerns from ultimate concern; that is, only that concern or concerns in a culture which function in terms of ultimacy are considered religious. Only that concern which is ultimate has the character of religion. It is all too apparent that on this analysis there is no such thing as an irreligious or nonreligious culture [...]. The analysis means only that there are elements of ultimacy which define the character of a culture, that some aspects of culture may be genuinely ultimate while others are not. In this sense religion is the substance of culture [...]."

${ }^{7}$ Hier ist zu bemerken, daß meiner Meinung nach kein Grund besteht, von ,Kultur" und ,Zivilisation" zu sprechen, wie das jedoch oft geschieht, indem man die eine mit den gestigen Aktivitäten verbindet, während die andere eine höhere Entwicklung auf dem materielltechnischen Lebensgebiet aufzeigen soll. Doch sind beide Begriffe in dieser Weise inhaltsmäßig nicht zu trennen. Über deren historische und semantische Bedeutung cf. Kroeber, 11 ff., besonders $19 \mathrm{ff}$; auch Tonnelat 73. Über die historische Entwicklung des Begriffs, ,Zivilisation" hat sich eingehend Febvre, $1 \mathrm{ff}$., besonders, 45, geäußert. Die Trennung dieser Begriffe lehnt auch Behrendt, 23, ab, der sich auf Woodard beruft.
} 
hang kultureller Äußerungen. Mir scheint, daß wir jetzt klarer erkennen, warum wir uns mit dem Thema unseres Symposiums beschäftigen. Es enthält die Forderung, die Stellung und Rolle der Religion im dynamischen kulturellen Prozeß sowohl in positiver als auch in negativer Hinsicht festzustellen. Das Schwergewicht ist jedoch auf den soziologischen Aspekt der Religion zu legen. Dessen besondere Aufgabe ist es, sich nicht nur mit Erscheinungen zu beschäftigen, wie sich die dynamischen religiösen Erlebnisse in statische Institutionen verwandeln, sondern überhaupt deren größere Zusammenhänge mit den sozialen Veränderungen aufzuzeigen.

2. Nach diesen drei Präambeln können wir uns der Hauptfrage unseres Symposiums zuwenden: der Einbeziehung religiöser Erscheinungen in das soziokulturelle Leben. Die Frage muß zugespitzt folgendermaßen lauten: Ist die Religion ihrem Wesen nach eine dynamische Erscheinung, die nur sekundär zu einer statischen Institution wird und damit einen stabilisierenden und konservierenden Faktor innerhalb der sozialen Strukturen bildet? Oder ist die Religion im Gegenteil in ihrer primären Auswirkung stets mit statischen und stabilen Formen verbunden, die allerdings in sich gewisse Bewegungen, d.h. dynamische Abläufe, enthalten und vertragen können? Mag das Problem, das diese beiden Fragen enthalten, auch im Mittelpunkt stehen, so sind diese Fragen in Wirklichkeit nicht vorrangig. Ins Zentrum der Überlegungen muß die Frage nach den Gründen, Faktoren und Motiven, die die Beziehungen zwischen dem dynamischen und dem institutionellen religiösen Leben bestimmen, gerückt werden. Wenn man sich des oben Gesagten bewußt bleibt, daß der Mensch ein dynamisches Wesen ist, kann man über Dynamik und Institutionalismus im religiösen Leben nur bedingt sprechen. Das heißt, einer solchen Einteilung fehlt der Charakter einer normativen Kategorie. Das führt praktisch zu Schwierigkeiten, wie sie sich auch in den einzelnen Beiträgen dieses Symposiums widerspiegeln. Einige sehen in den dynamischen Prozessen wesentliche Züge auch der religiösen Institutionen und deren Lebensformen, andere wiederum erblicken in den dynamischen Prozessen einen besonderen Ausdruck der stabilen institutionalisierten Traditionen. Ungeachtet dieser Schwierigkeiten ist und bleibt das Hauptproblem, die Motive und Faktoren zu klären, die den Übergang dynamischer Prozesse in Institutionen und umgekehrt bestimmen.

Im folgenden werden wir uns zwei typischen dynamischen Prozessen zuwenden, wie wir sie im soziokulturellen Leben feststellen können, dem 
sogenannten friedlichen, d.h. allmählichen Prozeß der Veränderung und dem revolutionären Unwandlungsprozeß.

Seit Linton 1954 sein Werk ,,Tree of culture“ veröffentlichte, pflegt man von drei Phasen der Kulturentwicklung zu sprechen. Es handelt sich um ein Evolutionsschema, das unter dem Einfluß der Naturwissenschaften entstanden ist. Unser Zeitalter wird dabei als dritte Phase in der Kulturentwicklung angesehen. In ihr findet eine Loslösung von der Abhängigkeit von den Naturkräften statt, und die ganze Aufmerksamkeit wird ,,social, political and economic fields" zugewendet ,We are now in the early phases of this third period and are only beginning to explore the potentialities which it offers for developments in our culture outside technology, particularly in the social, political, and economic fields. " ${ }^{8}$ Diese dritte Phase, d.h. also unser Zeitalter, wird daher auch als typisch dynamisch bezeichnet ${ }^{9}$.

2.1. Die dynamische soziokulturelle Situation wirft die Frage auf: Welchen Platz nimmt hier die Religion ein? Vielmehr: wie fügt sich die Religion in den dynamischen kulturellen Prozeß ein? Die Soziologen sind sich darüber einig, wie immer sie die Entstehung und das Wesen der Religion auch erklären, daß die Religion das soziale und kulturelle Leben bestimmt oder zumindest mitbestimmt. Der heutige Mensch steht ebenso wie der alte Ägypter vor dem selben unlösbaren Problem, nämlich vor dem Tod. In dieser Hinsicht hat sich die Situation des Menschen nicht geändert, zu welcher sozialen Struktur er auch gehören mag.

Es lohnt sich, hier einen der heutigen einflußreichen Soziologen zu Worte kommen zu lassen, der in seinen Überlegungen auch der Religion einen bedeutenden Platz eingeräumt hat. Das ist Topitsch. Sein Gedankengang ist folgender: , Immer wieder bestätigt sich Einsicht, daß elementare lebenspraktische und emotionale Bedürfnisse unsere Auffassung der Welt, des

\footnotetext{
${ }^{8} \mathrm{Cf}$. Lindton 48 . Er sagt weiter: ,It is safe to predict that even two or three centuries from now, such social inventions as modern-type Capitalism, Fascism, and Communism will be regarded as primitive experiments directed toward the adjustment of modern society to modern technology" (ib.).

${ }^{9}$ Die These von Linton hat auch Behrendt, 51 ff., nur wenig modifiziert übernommen. Über diese dritte Phase sagt er in klaren Worten: ,Eine entschiedenere und umfassendere Dynamik als je zuvor, im Sinne einer Entwicklung vom historisch Gegebenen weg in der Richtung auf bisher Unrealisiertes, ja, Unbekanntes und Unvorstellbares. In diesem Sinne, in dieser Perspektive, sind wir berechtigt, die Unterscheidung zwischen statischer Vergangenheit und dynamischer Gegenwart zu machen."
} 
eigenen Ich, der Erkenntnis und des Göttlichen von Grund her bestimmen. Das Hängen am Leben und die Angst vor dem Tod, das Begehren nach Glück und die Flucht vor dem Leid - das sind die Wurzeln, aus denen der primitive Mythos ebenso hervorsprießt wie die subtilste Spekulation. [...] Alle diese Faktoren sind von größter Wichtigkeit für die Vorstellungen, die sich der Mensch vom 'Göttlichen' macht. Es ist der Inbegriff des Freiseins von Tod und Leid, der Fülle des Lebens, des Glücks. Es ist das Wirkungskräftige, Mächtige, Erhabene und Majestätische."10 Diese sind die Umstände, die religiösen Vorstellungen bestimmen. Diese sind notwendig in einem von verschiedenen Faktoren bedrohten Leben. Man kann auch sagen, daß es sich um die Bejahung des Lebens dieser Welt handelt, jedoch unter der Bedingung, daß dahinter ein göttlicher Wille steht. Der Mensch kennt diesen zwar nicht, akzeptiert ihn aber. Topitsch fährt fort: ,Die Menschen haben sich allerdings nicht immer begnügt, ihnen unerreichbare Vollkommenheiten in den Bereich des Göttlichen zu projizieren, sondern sie haben oft auch versucht, dieser Vollkommenheiten trotz aller Hindernisse selbst teilhaftig zu werden. So durchzieht das Motiv der Selbstvergottung oder Gottwerdung die gesamte Geistesgeschichte, ja es reicht wohl bis in prähistorische Vorzeit zurück. Auf den verschiedensten Wegen etwa denen der Magie, der Mystik, der Spekulation - hat man danach getrachtet, sich göttliche Unsterblichkeit, Leidlosigkeit, Lebensfülle und Glückseligkeit zu verschaffen, aber auch die Macht und das Wissen der Gottheit. Vor allem die Vorstellung einer leidlosen und unzerstörbaren, vom vergänglichen Leibe loslösbaren und wesensverschiedenen Seele wurde häufig im Sinne einer solchen Selbstillusionierung verwendet."11 Hier kommen wir zum Kern seiner Ansichten, zur Gottwerdung des Menschen. Wie hat er sich das vorgestellt? Er findet, daß die Geschichte der Menschheit drei Formen aufweise, wie sich die Gottwerdung abgespielt habe, d.h. die Versuche, die zuvor erwähnten Unvollkommenheiten des Lebens, seine Mängel und den Tod zu überwinden ${ }^{12}$. Da ist erstens die in

10 Topitsch 1973, 9 f.

11 Ib. 11.

${ }^{12}$ So auch ib. $37 \mathrm{f}$.: ,,Drei Wege haben sich gezeigt, auf denen der Mensch die Gottwerdung als Überwindung des Realitätsdruckes vollziehen wollte, und alle drei sind in Vergeblichkeit ausgemündet: das bewußt-bewußtlose Innesein der Einheit von Âtman und Brahman, das Gottwerden des göttlichen Geistes durch Vermittlung des endlichen Geistes und die Aufhebung der Entfremdung durch die proletarische Revolution. Diese Vergeblichkeit ist durch die Voraussetzungen aller dieser Gedankengänge bedingt. Wird die göttliche Wesenheit durch ihre 
Indien in vielen philosophischen Spekulationen und Kulten anzutreffende Tendenz, zur Identität des Gott-Ich (Brahman-Atman) und damit zu einer Abwendung von der Realität zu gelangen. Zweitens gibt es das in antiken Mysterien bekannte Bestreben, die Wirklichkeit durch das Bewußtsein zu überwinden, daß die Seele des Menschen tatsächlich in einer anderen Welt zu Hause sei. Hier werde sie nur gefangen gehalten. In den Mysterienkulten habe sie die Möglichkeit, in ihren göttlichen Ursprung zurückzukehren. Von den Mysterienkulten hat diese Idee eine Weiterentwicklung in verschiedenen Zügen des Christentums erfahren. Drittens handelt es sich um das Versprechen des Marxismus, den empirischen Menschen und sein Leben so zu verändern, daß er vollständig vom Zwang der Realität befreit werde (Aufhebung der Entfremdung). Topitsch selbst gibt jedoch zu, daß keiner dieser Wege den Menschen dahin führe, wonach er sich sehne, nämlich, sich vom Zwang der Wirklichkeit zu befreien.

Wenn wir zum eben genannten Zitat von Topitsch zurückkehren, so muß man zugeben, daß die beiden darin erwähnten Vorstellungen, die Versuche, die Sehnsucht nach einem besseren Leben in eine göttliche Welt zu projizieren oder selbst in dieser Welt göttlich zu werden, oder wenigstens Teilhaber am Göttlichen zu sein, die Menschheit ununterbrochen begleiten.

Der im traditionellen theologischen Denken aufgewachsene und zumeist auch historisch und philologisch orientierte Religionswissenschaftler wird diese Ansichten zurückweisen, oft nur aus ideologischen Gründen ohne sachliche und kritische Prüfung. In diesen und ähnlichen Ansichten findet man gewöhnlich, wenn nicht einen direkten marxistischen Negativismus, so jedenfalls eine unmißverständliche Säkularisierung. Damit wollte man sagen, daß eine im Säkularismus begründete Ansicht niemals zu einem richtigen Verständnis religiöser Erscheinungen gelangen kann. Es bedarf keines Scharfsinns, um zu verstehen, daß Aussagen, die durch eine Ideologie begründet werden, durch Aussagen, die in einer anderen Ideologie (in diesem Fall einer theologischen) begründet sind, nicht sachlich bezweifelt

\footnotetext{
Unvergleichbarkeit mit allem Endlichen definiert, dann ist sie kraft eben dieser Definition dem Menschen unerreichbar. Wird sie in die Endlichkeit hineingezogen, dann wird sie selbst zum werdenden, leidenden Gott; erst recht bleibt dann der Mensch dem Realitätsdruck unterworfen, und die Gottwerdung bleibt ein leeres Wort oder ein bloßes Versprechen für die Zukunft. Dies letztere gilt besonders dort, wo man die Heraufkunft eines 'vergotteten', nicht mehr entfremdeten Menschen auf Erden erhofft."
} 
oder entkräftet werden können, wobei das Niveau oder die Bedeutung dieser Ideologie ganz außer Betracht bleibt. Topitsch lehnt mit großer Bestimmtheit diese beiden in verschiedenen Ideologien begründeten Ansichten über die Gottwerdung des Menschen ab. Die religiöse Version verwirft er deshalb, weil sie eine Darstellung der Schwierigkeiten des Lebens in die göttliche Welt projiziere. Eine solche Spekulation führe ins Leere und zu Widersprüchen, daher könne der heutige Mensch sie nicht akzeptieren ${ }^{13}$. Aber eine andere Möglichkeit, die damit rechnet, die Bedürfnisse des Menschen zu befriedigen und ihn im Rahmen des sozialen Lebens zu befreien, sei auch nicht gegeben, da sie die psychophysische und soziale Struktur der Existenz des Menschen nicht überwinden könne ${ }^{14}$.

In diesen Ansichten hat die Religion überhaupt keinen Platz. In unsere heutige soziale Situation paßt sie nicht hinein. Hier ist zu fragen, ob man dadurch, daß man die Bemühungen der Religion, dem menschlichen Leben einen Sinn zu geben, verneint und sie für illusorische Versuche erklärt, dem Zwang der Realität auszuweichen, nicht zu der so oft berufenen Leere kommt. Das hat auch der Vertreter dieser Ansicht erkannt: „Steht aber nicht auch derjenige, welcher alle diese - zum Teil gefährlichen - Illusionen durchschaut und verabschiedet hat, vor dem Leeren? Das mag sein, wenn wir darunter den Verzicht auf das 'absolute Glück' verstehen. Doch ist es nicht vielleicht das höchste uns erreichbare Glück, wenn wir lernen, mit dem Unglück zu leben?"15 Ähnliche Gedanken über Stellung und Rolle der Religion in der Situation des Menschen von heute finden sich auch in

${ }^{13}$ Ib. 14: ,,Der Mensch hat also unter dem Druck der Tatsache, daß die Beschaffenheit der Welt und seiner selbst als einer zu dieser Welt gehörenden psychophysischen Organismus vielen seiner elementaren Triebe, Wünsche und Wertungen nicht oder nur ungenügend entspricht, häufig Wesenheiten erphantasiert oder erdacht. So ist sie auch für den Menschen undenkbar und unerreichbar. Damit führt diese ganze Spekulation ins Leere, und der Wunsch nach Kommunikation oder Identifizierung mit einer solchen Gottheit endet im Widerspruch." ${ }^{14}$ Ib. 15: „Nicht besser steht es dort, wo der Mensch in der empirischen Realität seine 'Vergöttlichung' anstrebt, das heißt: die Befriedigung jener Triebe und Wünsche, die ihm unter den gewöhnlichen Bedingungen seines Daseins als physisches und soziales Wesen versagt bleibt. Sicherlich ist eine Verbesserung unserer Lebenssituation durch Veränderung der naturhaften und sozialen Welt in gewissem Ausmaße möglich und auch wünschenswert; doch stößt sie auf Schwierigkeiten, die nicht nur in den äußeren Verhältnissen liegen, sondern in erheblichem Grade auch im Menschen selbst."

${ }^{15} \mathrm{Ib}$. Cf. auch ib. 38 . Um jedoch die Ansicht von Topitsch noch besser zu verstehen, muß man den Gesamtzusammenhang kennen, durch den sich diese einzelnen Gedanken in sein System einfügen. Cf. id. 1972, $13 \mathrm{ff}$. und $47 \mathrm{ff}$. 
den Ansichten vieler anderer Soziologen. So äußert sich Behrendt von der Freien Universität Berlin: ,,Solange der Mensch das Wirken der Naturgewalten nicht versteht und sich ihnen gegenüber hilflos oder doch schwach empfindet, anheimgegeben an übermächtigte Kräfte, von deren unberechenbarem Walten sein Wohl und Wehe, ja seine Existenz abhängt, muß er sich ganz spontan transzendentalen Mächten zuwenden, die, wie er glaubt, dieses Geschehen bestimmten. Diese Mächte stellt man sich fast unvermeidlich als Gottheiten vor, in Form von Menschen- oder Tiergestalten, oder doch in analogen, der menschlichen Erfahrung nachgebildeten Formen." ${ }^{16}$ Das bedeutet dann ,,eine Verlegung des Schwergewichtes von der Bemühung um die Verbesserung des menschlichen Wesens und seiner Welt von innen her zu einer Bemühung von außen her; oder: vom Seelenheil zu sozialen Institutionen. Und die Sphäre des zu Bessernden wechselt von der Beziehung zwischen dem Menschen und der Gottheit zu der Beziehung zwischen dem Einzelnen und der Gesellschaft. Man glaubt jetzt a) an die Wichtigkeit des irdischen (individuellen) Glücks anstatt des Seelenheils; b) an die Realisierbarkeit des Glückes nicht so sehr durch innere Vervollkommnung (Kontemplation, Askese, mystische Entrückung, usw.), als durch institutionelle Veranstaltungen - also an die Realisierbarkeit des Glückes als einer 'sozialen Aufgabe'. Es setzt sich also geradezu eine Art von Glauben an die Erlösungskraft der Gesellschaft durch - an die Fähigkeit des Menschen, wenigstens kollektiv seine Probleme zu lösen." 17

Das von den Soziologen geschilderte Bild ist im großen und ganzen richtig. Es ist nicht zu leugnen, daß in unserer dynamischen Zeit solch eine soziokulturelle Verschiebung stattfindet. An dieser Tatsache ändert nichts, daß in gewissen religiösen und selbst intellektuellen Kreisen, die sich von der Technokratie unserer Zeit unbefriedigt fühlen, ein gesteigertes Interesse an religiösen Fragen zu beobachten ist. Davon zeugen auch die Probleme der Entstehung neuer Religionen, wie sie anläßlich unseres Symposiums vom Jahre 1974 behandelt wurden ${ }^{18}$. Ebenso wird das indirekt bestätigt durch das besondere Interesse der Kirche an soziologischen Problemen. Damit sucht diese auch nach Unterstützung für ihre eigenen Interessen ${ }^{19}$.

\footnotetext{
16 Behrendt 62.

17 Ib. 65.

${ }^{18}$ Cf. Biezais 1975, $12 \mathrm{ff}$.

${ }^{19}$ Savramis stellt fest, daß das nicht nur eine Modeerscheinung sei, sondern daß die Kirche
} 
3. Ungeachtet des eben Gesagten und der behaupteten Schichtung ${ }^{20}$ und Mobilitä $t^{21}$ unserer heutigen Gesellschaft spielt die Religion weiterhin eine große Rolle. Aus dem vorhin Gesagten wird verständlich, daß sich im religiösen Leben das Interesse an transzendenten Erscheinungen verringert hat. Aus der Behauptung, daß die Religion eine soziokulturelle Erscheinung sei, folgt auch, daß sie denselben Gesetzmäßigkeiten unterworfen ist wie alle Erscheinungen dieser Art. Mit anderen Worten: mit den strukturellen Veränderungen in der Gesellschaft wandeln sich auch die religiösen Erscheinungen bzw. deren Ausdrucksformen.

In den Veränderungen von Gesellschaft und Religion sind zwei Typen, zu beobachten. Man kann von allmählichen und von revolutionären Veränderungen sprechen. Wenn man annimmt, daß die ersteren selbstverständlich sind, verdienen die letzteren mit ihrer gewaltsamen Vernichtung alter Lebensformen und Werte besondere Aufmerksamkeit. Es ist notwendig, zum Thema unseres Symposiums diese beiden Aspekte im Auge zu behalten. Im ruhigen und allmählichen Wandlungsprozeß des sozialen Lebens sind Stellung und Rolle der Religion eine andere als in einer revolutionären Situation.

3.1. Der ruhige Umwandlungsprozeß der Gesellschaft und die Rolle der Religion dabei sind, so scheint es, am meisten im Zusammenhang mit dem sogenannten Funktionalismus diskutiert worden. Es lohnt sich, darauf hinzuweisen, weil diese Theorie in verschiedenen Modifizierungen innerhalb der angelsächsischen Welt, ungeachtet der scharfen und ablehnenden Kritik, weiterhin dominierend ist. Zunächst ist hier auf einige Nebenumstände

sich zur Verbesserung ihrer schwierigen Situation der Soziologen bedienen möchte, die mit ihren Ansichten populär und autoritativ geworden sind. Cf. Savramis 119; besonders auch Luckmann $28 \mathrm{ff}$.

${ }^{20}$ Es ist hier nicht möglich, die Ansicht von einer Umschichtung in den Aussagen der Theoretiker eingehender darzustellen, wie wir sie vor allem bei Parsons 1940, 848 ff.; 1957, 102 ff.; Davis 1942, $313 \mathrm{ff}$.; 1945, $244 \mathrm{ff}$.; Moore $16 \mathrm{ff}$. u. a. antreffen. Gegen diese Theorien der Funktionalisten hat sich auch eine scharfe Kritik erhoben, z. B. Buckley 1958, $369 \mathrm{ff} . ; 1963,799 \mathrm{ff}$., auch Tumin $391 \mathrm{ff}$., besonders 394 . Eine gute Übersicht über diese Diskussion gibt Wiehn 1968, $81 \mathrm{ff}$, zu beachten ist auch $115 \mathrm{ff}$. Er hat auch selbst zu dieser Diskussion beigetragen, cf. Wiehn 1975, 9 ff. Eine Loslösung vom Standpunkt der Funktionalisten ist bei Dahrendorf, $12 \mathrm{ff}, 31 \mathrm{ff}$., zu beobachten, der den Machtfaktor für primär hält. Ein Kennenlernen dieser Ansichten ist notwendig, denn wenn man die Religion als soziokulturelle Erscheinung betrachtet, ist sie organisch in die Umschichtungsprozesse der Gesellschaft eingebettet.

${ }^{21}$ Als grundlegendes Werk ist Sorokin, $133 \mathrm{ff}$., zu nennen, betreffend Skandinavien Carlsson, 1958, $172 \mathrm{ff}$. Cf. auch Mayer in Wiehn, 1975, $122 \mathrm{ff}$, , und Geiger $24 \mathrm{ff}$. 
hinzuweisen, die aus den Diskussionen mit der Zeit verschwunden sind. Vor allem kann man von Funktionalismus nur unter bestimmten Voraussetzungen sprechen, denn in Wirklichkeit besteht unter den Vertretern dieser Theorie keine Einmütigkeit, weder in bezug auf die formale Begründung der Theorie selbst noch über ihre praktische Anwendbarkeit. Der Funktionalismus ist zu einem Terminus zur Bezeichnung verschiedener, schwer zu vereinigender Ansichten in der Soziologie, Ethnologie, Sozialanthropologie usw. geworden ${ }^{22}$.

Beim zweiten Umstand, der hier zu beachten ist, handelt es sich darum, daß diese Theorie ihrem Wesen nach unhistorisch ist. Es grenzt an Ironie, daß sie aus ihrem geschichtlichen Zusammenhang gerissen wird. Gewöhnlich wird sie mit Malinowski und seinem ideellen Nachfolger RadcliffeBrown verbunden. Die Grundlage dieser Theorie ist eine holistische Kulturauffassung, gleichgültig, ob sie formal als ,,culture“ (Malinowski) oder ,,social structure“ (Radcliffe-Brown) bezeichnet wird. Der Holismus ist unter dem direkten Einfluß der Biologie entstanden und als deren Erbteil übernommen worden. Er steht im Zusammenhang mit der heftigen Diskussion über die mechanische und holistische (Vitalismus) Lebensauffassung am Ende des vorigen und zu Anfang dieses Jahrhunderts. Hier tat sich seinerzeit an erster Stelle Driesch hervor. Ausgehend von den Forschungen auf biologischem Gebiet, entwickelte er ein ganzes philosophisches System - die Philosophie des Organischen ${ }^{23}$-, was bei den heutigen angelsächsischen Funktionalisten in Vergessenheit geraten ist. (Darin besteht die Genugtuung der Forschung - etwas schnell zu vergessen, um es nach einiger Zeit mit Enthusiasmus aufs neue zu entdecken!)

3.2. Welche Rolle spielt die Religion im Konzept der heutigen holistischen Kultur? Rein formal kann man darauf antworten, daß sie im Begriff Holismus selbst ausgedrückt ist. Die Religion ist in die Gesamtheit der Kul-

${ }_{22}$ Über die unklare Terminologie siehe Jarvie 1965, 19; Steinbeck, Brigitte, 99. Etwas vereinfacht sagt Spencer 1: ,, The functional approach is at base simple; it seeks to do no more than assay the place of a particular element of culture or societal institutions in relation to other elements."

${ }^{23}$ Cf. Windelband 595 f.; näher über Drieschs Begriff des Ganzen bei Heinichen $453 \mathrm{ff}$.; auch Biezais 1939, 16 ff.; Martindale 154; Steinbeck, Brigitte, 100; und die scharfe Kritik von Hayek $57 \mathrm{ff}$; Hempel $277 \mathrm{f}$. Eine theoretisch motivierte Kritik bei Agassi $244 \mathrm{ff}$. Cf. auch die kritischen Anmerkungen gegen den Gebrauch des Ganzheitsbegriffes in den sozialwissenschaftlichen Arbeiten von Schlick 213: „'Ganzheit’ ist fast zu einem Zauberwort geworden, von dem man Befreiung aus allen Schwierigkeiten hofft." 
tur eingeschlossen, gleichgültig, mit welchem Synonym man diese bezeichnet - als Ganzheit, Harmonie, System, Struktur, Organismus, integrale Einheit u. a. Die Einheit der holistischen Kultur ist vor allem dadurch bestimmt, daß sie die biologischen Bedürfnisse des Individuums befriedigt, welcher sozialen Struktur dieses auch immer angehören mag. Tatsächlich ist damit das Bestehen der sozialen Einheit motiviert und bestimmt. Man könnte vielleicht erwarten, daß die Funktionalisten zwischen den sogenannten biologischen und den geistigen Bedürfnissen einen Unterschied machen. Das tun sie nicht. In der traditionellen Terminologie sind die sogenannten höheren - geistigen - Bedürfnisse (needs) nur Derivative der biologischen Erfordernisse. Im den Umstand, daß sie durch die bestehende soziale Ordnung gesichert werden, erblicken die Funktionalisten daher auch den Sinn des sozialen Systems. Damit haben wir eines der Probleme unseres Symposiums berührt, nämlich die Institutionen.

Wenn wir uns nun der Religion selbst zuwenden, muß sich unsere Aufmerksamkeit auf zwei im Mittelpunkt stehende Momente richten: es sind dies Mythos und Ritus. Aus der Darstellung der Grundposition der Funktionalisten ist bereits klar geworden, daß man keine theoretischen Erörterungen über Mythos und Ritus erwarten kann. Sie könnten nur pragmatischen Charakter haben. Verständlicherweise distanzieren sich daher die Funktionalisten von der bekannten Erklärung der Mythen als symbolische Formen religiöser Erlebnisse. Sie messen dem Mythos eine andere Bedeutung zu - nämlich eine soziale ${ }^{24}$. Indem die Mythen über die ethische Norm, die politische Macht, die gesellschaftliche Ordnung u.a. Grundlagen in der übernatürlichen mythischen Sphäre Mitteilung machen, verhelften sie dazu, die Stabilität dieser sozialen Institutionen zu sichern. Der Mythos ist die außerempirische Motivierung der statischen Gesellschaft. Dennoch konnte es den Funktionalisten nicht verborgen bleiben, daß in den Mythen ab und zu von Ereignissen aus einer bestimmten Zeit die Rede ist. Daher bemerkten sie sogleich, daß es sich nicht um eine Zeit im üblichen Sinne handeln könne, sondern um einen Begriff außerhalb der

\footnotetext{
${ }^{24}$ Malinowski 1931, 640 f:: ,The function of myth is to strengthen tradition and to endow it with a greater value and prestige by tracing it back to a higher, better, more supernatural and more effective reality of initial events." Noch deutlicher 1948, 117: ,Thus on the one hand the reality of myth lies in its social function; on the other hand, once we begin to study the social function of myth, and so to reconstruct its full meaning, we are gradually led to build up the full theory of native social organization."
} 
historischen Zeit. Schon damals sei die Ordnung, wie sie jetzt in der Gesellschaft anzutreffen ist, bestimmt worden. Damit erschöpfe sich die Rolle des Mythos in der Religion.

3.3. Wenn wir uns nun dem anderen im religiösen Leben vorkommenden Aspekt zuwenden, dem Ritus, empfiehlt sich der Hinweis auf die Gedanken von Radcliffe-Brown. Die Religion überhaupt und besonders der Ritus im weitesten Sinne garantieren eine integrale Gesellschaft, in seiner Terminologie ,,social structure“. Auch er stützt sich auf das Modell eines biologischen Organismus: ,To turn from organic life to social life, if we examine such a community as an African or Australian tribe we can recognise the existence of a social structure. Individual human beings, the essential units in this instance, are connected by a definite set of social relations into an integrated whole. The continuity of the social structure, like that of an organic structure, is not destroyed by changes in the units. Individuals may leave the society, by death or otherwise; others may enter it. The continuity of structure is maintained by the process of social life, which consists of the activities and interactions of the individual human beings and of the organised groups into which they are united. The social life of the community is here defined as the functioning of the social structure."25 Damit der Organismus funktionieren könne, müßten auch die physiologischen Prozesse im Einklang mit gewissen Gesetzen ablaufen. Ebenso sei es auch im sozialen Leben. Die Gesellschaft könne nur bestehen, wenn die einzelnen Elemente ihrer Struktur richtig funktionierten. Hier habe auch die Religion ihren Platz. Sie sei eines der Elemente, die das Funktionieren der Gesellschaft und damit ihr Bestehen garantierten ${ }^{26}$. Es haben Akzentverschiebungen vom Mythos auf den Ritus stattgefunden. Die Funktionen der Religion seien nicht in den symbolischen Vorstellungen und Aussagen der Mythen zu suchen. Im Gegenteil - ursprünglich sei der Ritus. Wie versteht Radcliffe-Brown den Ritus? Die Riten sind ,,regulated symbolic expressions of certain sentiments. Rites can therefore be shown to have a specific social function when, and to the extent that, they have for their effect to regulate, maintain and transmit from one generation to an-

25 Cf. Radcliffe-Brown 1969, 180.

26 Ib. 177: ,As a general formula (for whatever such a formula may be worth) it is suggested that what is expressed in all religions is what I have called the sense of dependence in its double aspect, and that it is by constantly maintaining this sense of dependence that religions perform their social function." 
other sentiments on which the constitution of the society depends. I ventured to suggest as a general formula that religion is everywhere an expression in one form or another of a sense of dependence on a power outside ourselves, a power which we may speak of as a spiritual or moral power." 27 Mit Hilfe der Riten werde für das Entstehen gewisser Gefühle gesorgt. Es handele sich um Gefühle, die die Überzeugung von der Notwendigkeit und Vollwertigkeit der bestehenden Gesellschaft hevorrufen und stärken. Tatsächlich sei das die einzige Aufgabe der Riten. Man greife auf sie insbesondere dann zurück, wenn die soziale Struktur in irgendeiner Weise gefährdet erscheine. Dann bekämen die rituellen Aktivitäten eine besondere Bedeutung. Das zeigt, daß die Rolle der Religion auf die praktische Erhaltung der sozialen Struktur reduziert wird. Das Individuum hat keine selbständige Bedeutung. Es wird vielmehr durch gewisse gesellschaftliche Normen unter Druck gehalten, denn es müsse der Tradition, der Religion, folgen, d. h.: , The primary basis of ritual, so the formulation would run, is the attribution of ritual value to objects and occasions which are either themselves objects of important common interests linking together the persons of a community or are symbolically representative of such objects. “ 28

Die Religion ist daher keine Erscheinung sui generis weder in psychologischer noch in soziologischer Hinsicht. Die praktische Funktion der Religion ist die Erhaltung der sozialen Struktur der Gesellschaft. Nun wird es verständlicher, warum es wichtig war, sich direkt mit der Auffassung der Funktionalisten zu befassen, denn diese bietet eine Begründung für die Institutionalisierung der Religion. Jetzt wird auch klar, warum die religiösen Institutionen in allen gesellschaftlichen Strukturen eine so große Rolle spielen. Damit enthüllt sich der eigentliche Sinn - nicht das Wesen - der Religion. An letzterem haben die Funktionalisten kein Interesse.

3.4. Es wäre naiv anzunehmen, daß die Funktionalisten nicht erkennen könnten, daß sich innterhalb der Gesellschaft und damit auch in der Religion als soziokultureller Erscheinung Veränderungen vollziehen. Das gilt jedenfalls für einen so aufmerksamen Erforscher empirischer Erscheinungen wie Malinowski. Er war einer von denen, die, um mit Jarvies Worten zu sprechen, den Mythos von der Feldforschung schufen. Er mußte auch die sozialen Veränderungen wahrhehmen. Es verhält sich nicht so, daß

${ }^{27} \mathrm{Ib} .157$., ,My suggestion is that in attempting to understand a religion it is on the rites rather than on the beliefs that we should first concentrate our attention" (ib, 155).

28 Ib. 151. Ähnlich auch Radcliffe-Brown 1948, 233 f. 
die Mythen, wie er behauptet, nur die bestehende soziale Organisation legitimieren, wobei er deren normative Motivierung in die mythische Vorzeit versetzt. Unter Berufung auf die Aussagen der Mythen und deren „Wirklichkeit" sind auch destruktive Bewegungen entstanden, die die bestehende soziale Ordnung nicht nur gestört, sondern sie auch haben zugrunde gehen lassen ${ }^{29}$. Wie überwinden die Funktionalisten diesen Widerspruch? Von der Position des Holismus aus können sie nur Veränderungen als normal annehmen, die sich in einer gesonderten sozialen Einheit vollziehen und nicht das ganze integrale System stören. Solche Funktionen müsse man dann als unecht oder vorübergehend bezeichnen. Ausgehend vom Modell des Organismus, sprechen die Funktionalisten dann von Dysfunktionen ${ }^{30}$. Eine Revolution ist damit in Wirklichkeit eine soziale Krankheit, an der der Organismus zusammenbricht und zugrunde geht. Auf Grund dieser biologisch bestimmten Konzeption könne man auch von endogenen und exogenen Faktoren im Prozeß der gesellschaftlichen Veränderungen sprechen $^{31}$. Die Religion gehöre in diesem Fall zu den endogenen Faktoren, da sie innerhalb einer bereits bestehenden Gesellschaft tätig werde ${ }^{32}$. Das

${ }^{29}$ Gewöhnlich wird hier das Material aus den verschiedenen Bewegungen des Cargo-Kults genommen: Jarvie 1970, 81 ff.; Worsley 146 ff.; Steinbauer 342 ff.; auch Biezais 1969, 27 ff.; aus Afrika Greschat $8 \mathrm{ff}$.

${ }^{30} \mathrm{Cf}$. Malinowski 1936, 62: ,, Those of us who believe in culture and believe in the value of religion, though perhaps not in its specific tenets, must hope that the present-day misuse of the religious apparatus for partisan and doctrinaire purposes is not a healthy development of religion, but one of the many phenomena in the pathology of culture which seem to threaten the immediate development of our post-war western society."

${ }^{31}$ So äußert sich Smith, 165, in seiner Kritik an den Funktionalisten: ,It is not necessary to point out the purely programmatic nature of this sample of major historical changes. But it is enough, I think, to justify our assertion that the exogenous paradigm is better able to cope with the vast range of types of change which we encounter in the historical record and observe around us. To ignore the impact of external intrusions, of diffusion, of the environment and of crisis, at every point in the vast majority of processes of change, is to allow theory to blind us to the evidence of history. In preferring history to evolution, we open the way to a genuine sociology of historical change, which the belief in immanent potentiality has till now barred." ${ }^{32}$ Auch Eickelpasch, 50, hat in seiner sehr bestimmten Kritik den endogenen und exogenen Faktoren des Funktionalismus zugewandt: ,Alle sozialen Einrichtungen, seien es Riten, Mythen, Scherzbeziehungen oder präferentielle Heiratsmodi, tragen nach Radcliffe-Brown zur Perpetuierung der Sozialstruktur bei. Alle Elemente sind fest integriert in das soziale System, heteronorne Partikel der Struktur. Als Faktoren des Wandels kommen sie daher nicht in Betracht. Nachdem Radcliffe-Brown einmal durch die Annahme der vollständigen Determiniertheit aller Elemente der Sozialstruktur die Möglichkeit endogenen Wandels ausgeschlossen hat, ist er gezwungen, jeden Wandel auf exogene Faktoren zurückzuführen." Doch ist seine Behauptung, daß Radcliffe-Brown u.a. die endogenen Faktoren der gesellschaftlichen 
funktionalistische Verständnis von der Religion ist eine unmißverständliche Reaktion auf die seinerzeit herrschende historisch-philologische Methode ${ }^{33}$.

4. In letzter Zeit haben sich, ausgehend von dieser Theorie, Ansichten gebildet, die der Religion einen autonomen Platz im soziokulturellen Gesamtzusammenhang zuweisen möchten, jedoch unter Beibehaltung der Grundposition des Funktionalismus. Hierbei haben sich besonders die angelsächsischen Forscher wie Berger, Luckmann, O'Dea, Yinger u. a. hervorgetan. In großen Zügen übernehmen auch sie die klassische These der Funktionalisten, daß es die Aufgabe der Religion sei, für die Stabilität der bestehenden Gesellschaft zu sorgen. Die Religion habe eine konservierende Rolle. Sie üben auf das Individuum einen Druck aus und erreiche damit, daß dieses sich mit der Gesellschaft, in der es lebt, solidarisiert. Kritiker haben bereits summarisch darauf hingewiesen, daß die Argumentation sich im Kreise dreht. Die Gesellschaft bedürfe, um zu bestehen, der Unterstützung durch die Religion. Die Religion wiederum werde als soziale Funktion betrachtet ${ }^{34}$. Gerade dieser Einwand wiegt am schwersten. Er

Veränderungen nicht kennen würden, nicht aufrechtzuerhalten. Die Funktionalisten als biologisch orientierte Vertreter des Evolutionismus betrachten auch Veränderungen in der Psychologie und Soziologie als Ausdruck organischen Lebens. Dahin gehört auch der Wandel der integralen Gesellschaft, wie das Smith, $50 \mathrm{ff}$., 130 s., $137 \mathrm{ff}$., ganz richtig erkannt hat, was er aber für eine ungenügende Erklärung der sozialen Veränderungen hält.

${ }^{33}$ Ich habe in dieser Übersicht nur die sogenannten Klassiker des Funktionalismus erwähnt. Aber bis zur letzten Zeit werden immer Versuche gemacht, neue Varianten des Funktionalismus auszuarbeiten. Darüber siehe Kolbs, 307, Hinweise auf Davis, Goode u. a. Goldschmidt, $40 \mathrm{ff}$., $131 \mathrm{ff}$., hat versucht einen ,,comparative functionalism“ auszuarbeiten. Diese Entwicklung hat Brigitte Steinbeck, 116f., auf folgender Weise charakterisiert: ,An dieser Stelle kann die vorausgeschickte These über den 'Funktionswandel' des Funktionsbegriffs auf dem Hintergrund des Materials, an dem sie abgelesen wurde, noch einmal formuliert werden. Der Funktionalismus entwickelte sich von einer Theorie zur Verfahrensweise, vom Erklärungsprinzip zum methodischen Mittel der Analyse. Die Funktion, die bei Malinowski als Wesenskonstante und Identifikationsmerkmahl sozialer Institutionen aufgefaßt wurde, bezeichnet jetzt lediglich die Art der Beziehung sozialer Elemente auf einen (hypothetischen) Zustand des ganzen Systems." Cf. auch weiter die kritischen Einwände von Bredermeier $173 \mathrm{ff}$.; Carlsson 1962, $229 \mathrm{f}$., Stinchcombe $91 \mathrm{ff}$; Jarvie 1965, $18 \mathrm{ff}$., besonders $28 \mathrm{ff}$; auch Desroche 1968, 73; Hempel 292.

${ }^{34}$ So Eickelpasch 53: ,Die Argumentation Radcliffe-Browns ist tautologisch. Einerseits hält er, wie wir sahen, den sozialen Wert eines Objektes für die Grundlage seiner Ritualisierung, andererseits leitet er im nachhinein aus der Tatsache der Ritualisierung eines Objektes dessen sozialen Wert ab. Die Riten sollen die Funktion haben, 'to establish certain fundamental social values'. Wenn aber, in der Terminologie Radcliffe-Browns gesprochen, der 'rituelle Wert' den 'sozialen Wert' eines Objektes konstituiert, dann kann letzteter nicht mehr zur Er- 
trifft sowohl die klassischen als auch die späteren Funktionalisten gleich hart. In den neuesten Forschungen hat man nun versucht, dieser Schwierigkeiten Herr zu werden ${ }^{35}$. In diesem Zusammenhang ist die Ansicht von Luckmann am bedeutsamsten. Er geht von dem Gedanken einer Weltanschauung des Individuums aus. Diese bilde sich durch die funktionellen Beziehungen zwischen dem Individuum und der Gesellschaft. Sie sei die anthropologische Basis der Religion ${ }^{36}$. Aber diese Feststellung allein befriedigt Luckmann nicht. Es ist eine zu allgemeine Erkenntnis, er will jedoch der Religion einen spezifischen Platz einräumen. Er spricht dabei vom ,sacred cosmos" des individuellen Glaubens im engeren Sinne. Jener zeichne sich durch drei verschiedene Merkmale aus: Allgemeinheit der Vorstellungen, d.h. generelle Bedeutung, ein Mysterium, das in einer vom Leben losgelösten Transzendens wurzele, Verwendung von Symbolen in den verschiedenen kultischen Handlungen. Damit erhält Luckmann ein dialektisches Schema. In diesem wird einerseits das spezifische religiöse Leben durch die drei genannten Momente charakterisiert, andererseits steht ihm das vorhin erwähnte Gedankensystem, d.h. die Weltanschauung, gegenüber. Diese letztere kann man auch als profane Welt bezeichnen. Auf diese Weise entspricht seine dialektische Gegenüberstellung dem uns bekannten Gegenüber von sakraler und profaner Welt. Luckmann hält an diesem Schema fest, weil es dann möglich sei, das Problem der religiösen Institutionen und deren Verhältnis zum Individuum

klärung des Ritus herangezogen werden." Cf. auch Scharf, Betty 73. Hier sei besonders an die Kritik des Funktionalismus erinnert, wie sie Yinger 1967, 58 ff.; ib. 1970, 89f., bietet.

${ }^{35}$ Diese Gruppe wird im Unterschied zum klassischen auch phänomenologischer Funktionalismus genannt. Doch ist damit ihr enger Zusammenhang mit dem Funktionalismus nicht zu leugnen. Cf. Scharf, Betty $74 \mathrm{ff}$.

${ }^{36}$ Luckmann 48f.: ,,Detachment from immediate experience originates in the confrontation with fellow men in the face-to-face situation. It leads to the individuation of consciousness and permits the construction of interpretive schemes, ultimately, of systems of meaning. Detachment from immediate experience finds its complement in the integration of past, present and future into a socially defined, morally relevant biography. This integration develops in continuous social relations and leads to the formation of conscience. The individuation of the two complementary aspects of Self occurs in social processes. The organism-in isolation nothing but a separate pole of 'meaningless' subjective processes-becomes a Self by embarking with others upon the construction of an 'objective' and moral universe of meaning. Thereby the organism transcends its biological nature [...]. In showing the religious quality of the social processes by which consciousness and conscience are individuated we identified the universal yet specific anthropological condition of religion." Ähnlich auch Berger 1963 a, $422 \mathrm{ff}$. Cf. auch Yinger 1970, $32 \mathrm{ff}$. 
besser zu verstehen und darzustellen. Eine so verstandene Religion ist universal, denn die zuvor genannten drei Momente charakterisieren jede Religion. Dagegen sind die Erscheinungsformen einer universalen Religion niemals universal. Diese manifestiert sich durch die empirisch gegebenen Institutionen. Diese wiederum werden nach Luckmann durch drei Umstände bestimmt. Erstens sind es die ökonomischen Verhältnisse, die überhaupt Vorbedingung seien. Nur dank diesen sei es möglich, daß sich eine besondere Gruppe von Vertretern einer Religion bilden könne. Zweitens handelt es sich um den Zusammenhang mit anderen sozialen Gruppen. Dieser fördere ein gesteigertes Bewußtsein von der Bedeutung und vom Wert der Gruppe und damit ihren Zusammenhalt. Dieser sei notwendig. So entstehe der Wunsch, ihn zu betonen und, falls nötig, auch zu verteidigen. Drittens seien die Vertreter einer Religion daran interessiert, ihre Ansichten zu präzisieren, d.h. die normativen Kategorien der religiösen Institution festzulegen. Nach Meinung von Luckmann ist dieser Institutionalisierungsprozeß durch die drei erwähnten Umstände bedingt und nicht zu verhindern. Zugleich mit der religiösen Institutionalisierung vollziehe sich eine solche nach denselben Prinzipien auch in den profanen Welt. Nach Luckmanns Terminologie geschieht das nach den sozialen Normen, wie sie in der Weltanschauung begründet seien. So findet er eine Bestätigung für sein vorhin erwähntes dialektisches Schema. Der dialektische Gegensatz dieser beiden Welten zeigt auch den Bruch zwischen ihnen auf. Heutzutage sind wir Zeugen dessen, daß die ursprünglichen Vertreter des sakralen Kosmos, die Hüter des religiösen Kultes, beiseite geschoben werden. Es ist ihnen nicht mehr möglich, ein ausgewogenes Bild der Einheit des sakralen Kosmos und der profanen Welt zu bieten, das der Mensch von heute akzeptieren könnte ${ }^{37}$. Im Endergebnis ist das eingetreten, was Yinger mit seinen Worten kennzeichnet: ,Religion itself may help to prevent a stratification system from being felt as an oppressive fact, as in the case of classic Hinduism or medieval Christianity, but it may not be able to do so in the face of competing value systems derived from non-religious sources or from some aspects of the religion itself. If a religion cannot 'explain away' the differences in income, power, and prestige on the basis of its own principles, it is less able to serve the function of integrating a society. Those who are most disadvantaged are particularly likely, under these circum-

${ }^{37}$ Cf. Luckmann $87 \mathrm{ff}$. 
stances, to desert the dominant religion and to accept some new religion, or proto-religion as the way to solve their problems." ${ }^{88}$

Die Entfremdung der institutionalisierten Religion von der sozialen Wirklichkeit bedeutet nicht, daß der Mensch von heute ohne sakrale Welt auskommen könnte. Das klingt bereits in den vorhin zitierten Worten von Yinger an. Doch sind es nur die praktische Notwendigkeit und die Lebenssituation, die dahin führen. Mit anderen Worten, es ist die gleiche bekannte pragmatische Motivierung der Religion wie bei den klassischen Funktionalisten. Praktisch ist der Mensch von Grund auf religiös. Doch unterscheidet sich seine soziale Situation und die damit motivierte Notwendigkeit der Religion z. B. bedeutend von der durch Tillich formulierten menschlichen Existenz angesichts der letzten Notwendigkeit (ultimate concern).

Luckmann benutzt sein dialektisches Schema auch zur Erklärung der dynamischen Erscheinungen. Der Bruch, von dem wir eben sprachen, zeige, daß sich in der statisch gewordenen und institutionalisierten Religion dank der Spaltung zwischen der Anschauung des sakralen Kosmos und der profanen Welt neue Aktivitäten religiösen Charakters entwickeln. Diese versuchen aufs neue, den entstandenen Bruch zu überbrücken. Diese jungen religiösen Bewegungen hätten die Tendenz, sich von den Institutionen abzuwenden, um die Forderungen zu befriedigen, die aus der verwandelten säkularisierten Umwelt erwachsen sind. Noch mehr: jede junge Bewegung trage die Tendenz in sich, statisch zu werden, in jeder statisch gewordenen Religion schlummerten schon die Wurzeln zu neuen Bewegungen.

Was hat man Luckmann mit seiner Auffassung erreicht? Wenigstens zweierlei. Er hat, obwohl er der Grundthese des Funktionalismus treu geblieben ist, dennoch gezeigt, daß die Funktion der Religion nur in einer statischen Gesellschaft auf die soziale Ordnung fördernd und konservierend einwirken kann. Das bedeutet, daß das Problem, das Radcliffe-Brown nicht lösen konnte und um dessentwillen er den Begriff der Dysfunktion oder der pathologischen Gesellschaft einführen mußte, hier seine Klärung findet. Die sogenannten Dysfunktionen entstehen in einer statischen Gesellschaft in Einklang mit dem dialektischen Prinzip. Es handelt sich um die Notwendigkeit des Individuums, seine persönliche religiöse Überzeugung mit den sozialen Normen der Gesellschaft in Einklang zu bringen. Die

${ }^{38}$ Yinger 1967, 68, auch id. 1970, 112. 
Theorie von Luckmann erlaubt es, noch mit einer anderen Schwierigkeit, der sich die Funktionalisten gegenübersehen, fertig zu werden. Entgegen der früher behaupteten praktischen Funktion der Religion bezeichnet Luckmann diese als eine Erscheinung sui generis. Der religiöse Mensch verliere, auch unter dem Druck der Gesellschaft stehend, niemals sein ,Ich“" Das bedeutet nicht nur, daß die soziale Umwelt auf ihn einwirkt, sondern auch umgekehrt, daß er stets schöpferisch die Institutionen der Gesellschaft beeinflußt. Mit anderen Worten, die religiösen Institutionen haben nur dann mit ihren Normen und Symbolen für das Individuum einen Sinn, wenn sie von ihm persönlich mitgebildet worden sind. Ich sagte einleitend, daß es der bezeichnendste Zug des Menschen sei, ein dynamisches Wesen zu sein. Ein solches bleibt er auch in jeder sozialen Situation. Wenn das Individuum nicht selbst im Normensystem der Gesellschaft einen Sinn erkennen bzw. ihm einen solchen erteilen und dessen Wertmaßstab nicht anerkennen würde, würde es in einer ständigen Situation der Sinnlosigkeit leben. Sobald auf Grund einer sozialen Veränderung (ökonomischer, politischer u. a. Art) ein solcher Zustand einzutreten droht, reagiert das Individuum darauf. Er sucht eine sinnvolle Rechtfertigung seines Lebens in den veränderten sozialen Verhältnissen. Das ist stets ein kreativer Prozeß. Hier wird auch der Unterschied dieser Auffassung zum traditionellen Funktionalismus sichtbar, der behauptete, daß die Gesellschaft das Individuum immer dazu zwinge, sich ihr zu unterwerfen, um das Gleichgewicht wiederherzustellen. In dieser Auffassung ist es eher das Individuum, das in einer gegebenen Situation kreativ auf die Gesellschaft einwirkt und deren Umwandlungsprozeß bestimmt ${ }^{39}$.

Ähnliche Gedanken wie Luckmann hat auch O'Dea geäußert, der ebenfalls vom Funktionalismus ausgeht und ihn modifiziert. Er hält im ganzen am Begriff der Tradition fest, indem er der Religion einen autonomen Platz neben solchen geistigen Aktivitäten einräumt, wie sie sich auf dem Gebiet von Kunst und Wissenschaft äußern. Doch ist er sich sehr deutlich der

\footnotetext{
39 Daß das nicht immer so ist, erwähnt auch Yinger, 1970, 111, in seiner systematischen Übersicht: ,When the 'established expectancies' of the members of a society are frustrated. This refers, not to some absolute level of need, but to the satisfactions that the members of a society have come to expect. When these are denied, those who feel frustrated may become more religious, in the manner of the Old Believers in Russia, or that of the Black Muslims of the United States or of the explosive Kitiwala movement in the Congo, which is a politicoreligious sect built around Christian symbols. But becoming more religious, in these cases, does not mean embracing more strongly a unifying religious tradition."
} 
großen Schwierigkeiten bewußt, die dynamischen Prozesse mit dem Institutionalismus im religiösen Leben in Einklang zu bringen. Von Luckmann kann man sagen, daß sein dialektisches Schema wenigstens einen Versuch darstellt, zu einer Art Lösung zu kommen. Das kann man von O'Dea nicht behaupten. Er hat sich damit begnügt, die bekannten fünf Dilemmas, wie er diese Widersprüche nennt, aufzuführen ${ }^{40}$.

Hier ist auch noch Yinger zu erwähnen, der sich in seinen vielen Arbeiten intensiv mit diesen Problemen beschäftigt hat. In der Gruppe der modernen Funktionalisten neigt er am ehesten dazu, in der Religion eine besondere Erscheinung zu sehen. Es scheint, daß sich seine Anschauung unter dem direkten Einfluß von Religionspsychologen gebildet hat. Er geht auch aus von der individuellen Situation des Menschen, in der dieser vor allen anderen Dingen auf die Frage nach dem Sinn seines Lebens antworten muß. Nur die Religion könne helfen, die Existenzangst und die Furcht vor dem Tode zu überwinden. Das vermag seiner Meinung nach die Wissenschaft nicht. Wer das behauptet, stelle der Wissenschaft Aufgaben, die ihr nicht zukommen, nämlich Werturteile auszusprechen ${ }^{41}$. Nach Meinung von Yinger bietet die Religion dem von Ängsten bedrückten Menschen Hilfe, bezeichnenderweise unabhängig davon, welcher sozialen Grupper er angehöre. Andererseits werde das Individuum durch die Zugehörigkeit zu einer religiösen Gemeinschaft, einer sozialen Einheit im Kampf gegen diese Ängste in seiner religiösen Überzeugung gestärkt $t^{42}$.

5. In den Ansichten dieser Wissenschaftler läßt sich, ungeachtet ihrer direkten Abhängigkeit vom Funktionalismus, dennoch eine bedeutende Weiterentwicklung erkennen. Das bezieht sich vor allem auf die Anschauung von der Rolle des Individuums. Besonders ist die Tendenz zu bemerken, der Religion ihren besonderen Platz innerhalb sozialer Entwicklung zuzuweisen. Doch ungeachtet dieses Wandels der Ansichten der Funktionalisten sind weiterhin die Mängel dieser Auffassungen deutlich sichtbar. Die Kernfrage, an der vorbeigegangen oder die offen gelassen wurde, ist die nach

\footnotetext{
${ }^{40}$ O'Dea 1964, 208; ,,Wir stoßen hier also auf ein Paradoxon: Die Institutionalisierung macht das Heilige ein wenig gewöhnlich. Diesem fundamentalen Paradoxon entspringen fünf Dilemmas. Mit anderen Worten: Die Religion braucht die Institutionalisierung, aber sie leidet auch darunter." Ähnlich 1961, 32; cf. auch id. 1970, 240 ff. Über die Ansichten von O'Dea hat sich Yinger 1961, $40 \mathrm{f}$., auch id. 1970, $235 \mathrm{f}$, näher geäußert.

${ }^{41}$,Belief in science is diffuse through the structures and cultural values of some societies rather than institutionalized as a distinctive religious pattern", sagt Yinger 1970, 195. Cf. auch ib. 12.

42 Cf. Yinger 1963, 42f.
} 
den Ursachen der religiösen Veränderungen. Es ist diesen Forschern gut gelungen aufzuzeigen, wie die sozialen Systeme mit der Suche des Individuums nach dem Sinn des Lebens in einem "sacred cosmos" zusammenhängen, aber sie können die Frage nicht beantworten, warum ein besonderer ,,sacred cosmos" gerade mit einem bestimmten sozialen System verknüpft ist oder, einfacher gesagt, warum sich besondere religiöse Vorstellungen, sowohl harmonische als auch unharmonische, je nach der gegebenen sozialen Umwelt, herausbilden. Diese Forscher können auf die Fragen antworten, wie diese Vorstellungen funktionieren und wie nicht, aber die Frage nach deren spezifischem Charakter und ihrem Zusammenhang mit gewissen sozialen Strukturen bleibt unbeantwortet ${ }^{43}$. Die zweite offene Frage ist die nach der religiös-sozialen Innovation und nach unvorhergesehenen Krisenfällen. Religiöse Reformen und revolutionäre Situationen kann man nicht mit Hilfe des holistischen Gleichgewichts und der Forderung nach Harmonie erklären ${ }^{44}$.

6. Wie wir gesehen haben, ist der soziale Wandel eine Erscheinung, der auch die Vertreter der Theorie einer statischen Gesellschaft Aufmerksamkeit widmen müssen. Sie begnügen sich allerdings gewöhnlich nur mit solchen Erklärungen, die ihrer Grundkonzeption entsprechen. Doch bleibt die Tatsache bestehen, daß sich neben der statischen Gesellschaft auch Bewegungen feststellen lassen, die man als revolutionär bezeichnen mu $3^{45}$. Auch sie bedürfen einer Erklärung. Diese kann sehr verschieden sein. Man kann es wie Steinbacher machen und von einem , unumgänglichen Kon-

\footnotetext{
${ }^{43}$ Sehr klar werden die Schwierigkeiten bei dieser Ansicht von Scharf, Betty, 82, erkannt und etwas zugespitzt formuliert: „But it leaves in the air the question of why a qualitatively different 'sacried cosmos' should form the apex of each system, and whether it will function to support any particular social order. On the other hand, if the aspect of the sacred as power is stressed, the grounds of belief in such a power seem to be only that it is part of a system of meanings which is external to the individual, since such a system can only be produced by the interaction of men in space and time [...]. Our authors have interesting things to say about the interaction of systems of ideas and social structures in modern industrial societies, and therefore about the function or dysfunction of these ideas in respect of the cohesion of various social groups such as family and state; what they do not show is that religious ideas have a necessarily stronger role to play in the maintenance of social groups than other kinds of ideas."

${ }^{44}$ In seiner allgemeinen Kritik des Funktionalismus hat Smith, $164 \mathrm{f}$, , besonders darauf hingewiesen.

${ }^{45}$ Erst in allerletzter Zeit haben sich die Theoretiker des Funktionalismus auch mit der Revolution als soziologischem Problem befaßt, wobei sie zur Bezeichnung solcher Veränderungen einen besonderen Begriff - ,,dynamic equilibrium" (Eisenstedt, Johnson, Smelser) - einführten. Eine Kritik dieses Versuchs lesen wir bei Smith $130 \mathrm{ff}$.
} 
flikt" sprechen, der in der dynamischen Natur des Menschen selbst begründet sei. Unter Berufung auf seine Forschungen über Aggressivität meint Lorenz, daß die Gründe für eine revolutionäre Konfliktsituation nicht immer in exogenen Verhältnissen zu suchen seien, sondern eher in der Natur des Menschen als eines biologischen Wesens lägen ${ }^{46}$. Diese lässe ihn in Konfliktsituationen geraten. Gestützt auf diese Erkenntnis, könnte man meinen, daß sich die revolutionären Bewegungen unaufhaltsam aus der Natur des Menschen entwickeln. Doch trägt diese Erklärung wenig zum tieferen Verständnis der dynamischen Erscheinungen bei. Sie ist zu summarisch.

Es ist nicht möglich, hier auf die vielen einander widersprechenden Ansichten über die revolutionären Bewegungen, wie sie in einer umfangreichen Literatur zu finden sind, einzugehen ${ }^{47}$. Hier seien nur einige typische Beispiele zur Beleuchtung unseres Problems erwähnt. Den Begriff Revolution zu definieren, ist sehr schwer, da er sich im Laufe der Geschichte gewandelt hat. Er wechselt auch abhängig vom Wertsystem, innerhalb dessen er gebraucht wird, bzw. von der Ideologie, der er angehört. Wir können hier nur auf eine Definition hinweisen, die in unserem Falle ausreicht. Smolik sagt, dass die Revolution als ,,die gewaltsame Dislokation des Zentrums der Macht aus der Hand einer gesellschaftlichen Klasse in die Hand einer neuen auf die geschichtliche Bühne auftretenden Klasse verstanden" ${ }^{* 48}$. Diese Definition ist zwar sehr allgemein gehalten, und es wird darin nur das durch die Revolution erreichte Resultat berücksichtigt. In Wirklichkeit ist der Prozeß viel komplizierter. Ich möchte meinerseits nur auf zwei Umstände hinweisen und diese durch einige Beispiele erläutern. Erstens handelt es sich um die Rolle des Individuums im revolutionären Prozeß und zweitens um die Charakterisierung des durch die Revolution erreichten Zustandes.

6.1. Die Rolle des Individuums läßt sich gut an Hand von Beschreibungen dynamischer Situationen im Alten und Neuen Testament studieren $^{49}$. Hier sei an die Rolle der Propheten in der von einer Priester-

${ }^{46}$ Cf. Steinbacher 208.

${ }^{47}$ Z.B. Desroche $1973,67 \mathrm{ff} ., 153 \mathrm{ff}$.

${ }^{48}$ Smolik 565. Eine andere Definition bei Smith 96.

${ }^{49}$ Wenn auch in letzter Zeit solche Gedanken gelegentlich mit Jesus verbunden werden, wird jedoch der soziologische Inhalt dieses Begriffs gewöhnlich durch theologische Spekulationen ausgehöhlt (Gerhardsson, Wingren u. a.). Gleich meinungslos ist auch der positive Versuch Stählis (cf. id. 138 ff.). 
hierarchie bestimmten israelitischen Religionsgemeinschaft erinnert. Die Analysen und Hinweise von Weber auf den charismatischen Charakter der Person des Propheten sind besonders bedeutsam, aber weithin bekannt, so daß sich ein nochmaliges Eingehen darauf erübrigt. Der Kampf der Propheten gegen die Priester des Kultes ist besonders heftig. Diese Auseinandersetzungen richteten sich gegen die von der Monarchie akzeptierte institutionelle kultische Gemeinschaft, deren Hüter die Priester waren. Die Polarisierung der Situation ist klar. Die Forderungen der Propheten sind auf eine Umwandlung des herrschenden religiösen und sozialen Lebens gerichtet. Das bedeutet, daß die Stellung der Propheten in der gegebenen sozioreligiösen Situation die von Revolutionären ist ${ }^{50}$. Ihre radikalen Forderungen nach Reformen sind in einer göttlichen Offenbarung begründet oder, um einen zeitgebundenen Ausdruck zu benutzen, in einem Befehl Jahves. Diese Offenbarung ist persönlich, d. h. sie findet außerhalb des offiziellen Kultes und der Kontrolle seiner Repräsentanten statt. Es ist nicht meine Absicht, hier die Situation der Propheten mit ihren vielen historischen und religiösen Aspekten darzustellen, sondern ich möchte nur auf die grundlegende Tatsache hinweisen, daß man es hier mit revolutionären Aktivitäten zu tun hat, die vom Individuum ausgehen. Es ist in diesem Falle unerheblich, welche psychologischen, soziologischen oder anderen Motive das Individuum dazu gebracht haben. Ebenso ist es eine ganz andere Frage, wieweit die Forderungen der Propheten realisiert wurden. Ich möchte hier besonders betonen, daß die Person des Propheten und seine Forderungen nicht in die soziale Umwelt aufzulösen sind, obwohl er innerhalb dieser als Individuum tätig ist.

Der Prozeß der historischen Entwicklung während zweier Jahrtausende

\footnotetext{
${ }^{50}$ Hier ist an die Aussage von Volz, 19, zu erinnern: ,Die alttestamentliche Religion, die Propheten-Religion, ist Wort-Religion, und dadurch steht die alttestamentliche ProphetenReligion im schärfsten Gegensatz zur Priester-Religion, zur Kult-Religion. PriesterReligion ist Opfer-Religion; der Priester bringt die Gaben der Menschen von unten hinauf zur Gottheit. Propheten-Religion ist Wort-Religion; sie bringt die Stimme Gottes von oben herab zum Menschen." Gestützt auf das Evolutionsschema des Positivismus und tief unter dem Einfluß der Schule „Myth und Ritual“ stehend, hat Berger, 1963, 944, in seiner Polemik gegen Weber versucht, diesen Gegensatz zu leugnen: ,,Various degrees of 'domestication' of prophetic elements within the cult occurred." Es ist selbstverständlich, daß die Propheten einer gewissen soziokulturellen Umwelt entstammen, aber ihre Tätigkeit zeigt, daß sie diese niemals akzeptiert haben, noch weniger sich einer solchen ,domestication“, wie Berger sie erkannt haben will, überlassen haben. Auf diese Weise, wie er das möchte, ist die von Weber festgestellte Rolle der charismatischen Person in den dynamischen Prozessen nicht abzulehnen.
} 
hat die Person Jesu mit einem undurchdringlichen Netz von Gedanken und Ansichten überdeckt, so daß es fast nicht mehr möglich ist, darunter die individuellen Züge seiner Person zu erkennen. Bezeichnend sind jedoch zwei Dinge. Die christliche Kirche und ihre Dogmatik haben sich geweigert, in Jesus einen Revolutionär zu sehen. Diejenigen, die das behauptet haben, sind gweöhnlich von der Kirche mehr oder weniger abgelehnte Nichttheologen gewesen. Dagegen haben mehrere jüdische Forscher Jesus als einen revolutionären Kämpfer seiner Zeit angesehen ${ }^{51}$. Entsprechend dem theokratischen Denken berief sich Jesus auf die ihm von Gott verliehenen Vollmachten zur Umwandlung des religiösen, sozialen und politischen Lebens. Sein revolutionäres Bewußtsein darf hier nicht mit seiner religiösen Motivierung verwechselt werden. Als Revolutionär, nicht als religiöser Denker, wurde er ausgeliefert und verurteilt. Dieser Standpunkt ist wiederholt von seiten jüdischer Forscher geäußert worden: ,, The thesis of the book is at variance with traditional views. It undertakes to prove that Jesus thought of himself as no more than the herald of an imminent material transformation of the world (the Kingdom of God), that his message was addressed to the Jews of his own time and to no one else, and that upon the failure of the Kingdom of God to appear he embarked on an altogether different course of action, which led to his violent death." ${ }^{62}$ Es ist selbstverständlich, daß solche Ansichten in der von der Kirche bestimmten Theologie keine Unterstützung fanden ${ }^{53}$. Aber ungeachtet dieser ablehnenden Haltung sind auch von dieser Seite in allerletzter Zeit Ansichten geäußert worden, die den revolutionären Charakter Jesu zugeben. Hier ist besonders der Professor der Theologie Smolik von der Universität Prag zu erwähnen. Er gibt zu, daß es mangels sicherer historischer Quellen nicht möglich sei, eine vollständige Biographie Jesu zu schreiben, doch sei das Material derart, daß es einige bezeichnende Züge in der Person Jesu erkennen lasse, die ihn als Revolutionär charakterisierten. Dazu gehöre das Bewußtsein Jesu von seiner Souveränität. Sie ist so groß, daß sie ,,ihm die

${ }^{51}$ Cf. Eisler 2, 57, $439 \mathrm{ff}$.

${ }_{52}$ Carmichael V.

${ }_{53}$ Auf der einen Seite steht Jesus als historische Person, der unter empirisch gegebenen soziokulturellen Verhältnissen hervorgetreten und tätig geworden ist, etwas anderes ist der Kultgott der christlichen Kirche, der sich zum Teil aus der historischen Person Jesu, zum Teil unter dem Einfluß anderer Kultgötter entwickelt hat. Über diesen Prozeß hat sich Bultmann, $191 \mathrm{ff}$, überzeugend geäußert; cf. auch Biezais 1963, 64. 
Relativierung der Autorität Moses und des Gesetzes ermöglichte" ${ }^{\text {"54 }}$. Ein anderer bezeichnender Zug Jesu ist seine ,,Solidarität mit Sündern, Diskriminierten, Gottlosen", die im Gegensatz zu der Auffassung vieler offizieller Repräsentanten der Religion seier Zeit stand. Diese Momente charakterisieren das revolutionäre Bewußtsein Jesu. In einem Punkt macht das traditionelle theologische Denken einen wesentlichen Unterschied ${ }^{55}$. Das Reich Gottes wird von ihm als eine ,,geistige Wirklichkeit", sowohl in psychologischer als auch in metaphysischer Hinsicht betrachtet. Das ist auch der Grund, weshalb es vom säkularisierten Standpunkt aus für eine Utopie gehalten wird. Gewiß, wenn man sich von ideologischen Hemmungen befreit, muß man Jesus als revolutionäre Gestalt betrachten ${ }^{56}$. Dieser Einsicht in die Wirklichkeit hat aber auch stets ein anderes besonderes Hindernis im Wege gestanden. Seit den Zeiten der Französischen Revolution war der Begriff Revolution in Europa stets mit einer säkularisierenden politischen Bewegung verbunden. Im Einklang mit unserer vorhin erwähnten Definition des Begriffs Revolution und den hier dargestellten Zügen in Jesu Leben ist es nicht schwer einzusehen, daß es sich bei der von Jesus ausgelösten Bewegung um eine Revolution handelt. Damit sind wir bei einem wichtigen Moment in der Erörterung unseres Problems angelangt, nämlich bei der Feststellung, daß Jesus als Individuum eine weltweite Bewegung, das Christentum, hervorgerufen hat.

Es ist kein Zufall, daß die heutzutage auftretenden sogenannten messia-

${ }^{54}$ Smolik 566. Er beruft sich auf Käsemann 208: ,, Jesus hat mit einer unerhörten Souveränität am Wortlaut der Tora und der Autorität des Moses vorübergehen können. Diese Souveränität erschüttert nicht nur die Grundlagen des Spätjudentums und verursacht darum entscheidend seinen Tod, sondern hebt darüber hinaus die Weltanschauung der Antike mit ihrer Antithese von kultisch und profan und ihrer Dämonologie aus den Angeln. Man kann das nicht konstatieren, ohne in lebhafte $Z$ weifel gegenüber dem so überaus gängigen Bilde des Frommen etwa gar aus dem Kreise der Anawim zu geraten, welcher Tag und Nacht die Schrift studiert und in ihr das Modell seines Weges als Gottesknecht oder leidender Messias beschrieben findet." ${ }_{55}$ Cf. Ib. 566; auch Stähli, 145 ff., mit dem inhaltslosen Begriff vom Reich Gottes.

${ }^{56}$ Die revolutionäre Wirksamkeit Jesu hat neulich auch Kamlah, 31, erwähnt, besonders 33: „,Gänzlich verschieden von der in die Welt ausgreifenden chiliastischen Revolution ist die innerreligiöse Revolution mancher Propheten des Alten Testaments und dann Jesu und des Paulus. Jesus protestiert dagegen, daß der Jude sein Verhältnis zu Gott durch gewissenhafte Befolgung des Moses-Gesetzes ins reine bringen will, und Paulus wiederholt diesen Protest, indem er hinzufügt, daß die Bereinigung der menschlichen Beziehung zu Gott nicht durch menschliches Handeln, sondern allein durch Gottes Vergebung in Christus geschieht." Siehe auch in diesem Zusammenhang, was Hauser, $241 \mathrm{f}$, über die Utopie sagt, und Jordan, 178f., über die säkularisierten Heilslehren. 
nischen Bewegungen die gleichen Vorgänge bezeugen ${ }^{57}$. Unabhängig davon, ob sie sich mit den politischen, sozialen und ökonomischen Verhältnissen der einheimischen Völker oder deren ursprünglichen religiösen Mythen oder gar mit Vorstellungen, die diesen fremd sind, verbinden, die die Christen mitgrebracht haben, stets entwickeln sie sich zu Unruhen und Aufständen $^{58}$. Gewöhnlich beobachten die Forscher den kollektiven Charakter solcher Bewegungen. Sie sprechen dann von sozialen Bewegungen. Einem aufmerksamen B eobachter jedoch fällt vielmehr in die Augen, daß sich diese Bewegungen um eine Hauptperson bilden. Gewöhnlich erscheint diese bereits zu Beginn der Bewegung. Daher wird sie von den Kolonialmächten zuerst isoliert und verfolgt ${ }^{59}$. Das Tatsachenmaterial darüber ist sehr umfangreich und eindrucksvoll.

Dieser Einblick in die revolutionären religiösen Bewegungen, die durch ihren dynamischen Charakter hervorstechen, macht deutlich, daß die Ansichten, die hauptsächlich nur die kollektive Seite betrachten, einseitig sind und die Erscheinungen nicht in ihrem ganzen Umfang berücksichtigen. Dies ist keine neue Erkenntnis, die wir hier gewonnen haben, sondern lediglich ein Hinweis darauf, daß über den dominierenden Ansichten der Funktionalisten in der Diskussion vergessen wurde, daß die ganze Zeit über auch

${ }^{57}$ Siehe den 7. Band unserer Serie, der mehrere Beschreibungen solcher Bewegungen nebst umfangreichen Hinweisen auf die entsprechende Literatur enthält.

${ }^{58}$ Reiches Material über diese Bewegungen findet sich bei Lanternari 1966, $37 \mathrm{ff}$.; Steinbauer $17 \mathrm{ff}$., u. a.

${ }^{59}$ Hier ist an das Schicksal von Simon Kimbangu zu erinnern. Es fehlt nicht an Beispielen, daß die einheimischen Mächte der neubegründeten Staaten in Afrika auch solche Führer verfolgen, z.B. Alice Lenshina (cf. Lanternari 1965, 105). Das geschieht, weil man deren Tätigkeit als revolutionär, staatsfeindlich empfindet. Über die Beziehungen religiöser und politischer Momente in diesen Bewegungen hat Talmon, Yonina, $125 \mathrm{ff}$., eine sehr instruktive Analyse gegeben; auch $145 \mathrm{f} .:$,, There is an even more marked affinity between these political movements and millenarism. Secular revolutionism shares with millenarism the apocalyptic element-like millenarism it looks forward to a total and imminent realization of its ideals. Millenarism shares with secular revolutionism its collective and terrestrial emphasis as well as the radical condemnation of the existing social order. Both the religious and secular versions of revolutionism reject gradual and peaceful improvement and prepare for a final decisive struggle. Millenarism has a more passive definition of the role of the follower, but, by and large, we find in both versions of revolutionism a potent merger between inevitability and freedom which assures the revolutionaries of their final triumph, yet endows them with some power to hasten or retard salvation. There is in addition a partial similarity in the conditions which bring about such movements. Like millenarism, secular revolutionism is brought about by a combination of severe deprivation, acute frustration and disintegration of primary groups." 
andere Meinungen geäußert und vertreten worden sind. Aus Raummangel können wir uns damit hier nicht näher befassen, sondern nur auf diese Tatsache verweisen ${ }^{60}$.

6.2. Wie ich bereits in einem anderen Zusammenhang erwähnt habe ${ }^{61}$, so hat auch Jarvie begründet festgestellt, daß zwischen der Person des Erweckers einer Bewegung und ihren Anhängern nicht immer Übereinstimmung besteht, weder in bezug auf die Intentionen, noch auf die Ziele. Vielfach bleibt die charismatische Person mit ihrer Tätigkeit und Verkündigung allein und ruft keinerlei Bewegung hervor, ein andermal scheint es, als ob die führende Person ihre wichtige Rolle verloren hat und von der Bildfläche verschwunden ist. In den Vordergrund geschoben haben sich dann die Anhänger der Bewegung. In diesem Zusammenhang präzisiert Jarvie seine Ansicht. Diese Variationen in den Beziehungen zwischen der charismatischen Person und den Anhängern der Bewegung sind mit der jeweiligen besonderen Situation zu erklären ${ }^{62}$. Diese Erkenntnis ist für unsere weitere Erörterung besonders wichtig.

Einen bedeutsamen Platz innerhalb der revolutionären Bewegungen nimmt auch eine andere Erscheinung ein. Um diese zu charakterisieren, müssen wir uns nochmals der biblischen Welt zuwenden. Als von den Propheten des Alten Testaments die Rede war, übergingen wir eine besondere, gut bekannte und oft erwähnte Gruppe, die nebiim (ekstatische Wüstenderwische), die zeitweise zu mehreren auftraten. Diese wurden von den Kultpriestern, d. h. von den offiziellen Repräsentanten der Religion, verfolgt. Was uns besonders interessiert, ist, daß ein nabi gewöhnlich keine Volksbewegung hervorgerufen hat. Dasselbe gilt auch für die israelitischen Propheten überhaupt. Das bedeutet aber ganz und gar nicht, daß deren For-

${ }^{60}$ Hier ist an die Theorien und Aussagen eines solchen Klassikers der Soziologie wie Thomas über die Rolle des Individuums in revolutionären Situationen zu erinnern: ,,Jene Art der aktiven Opposition gegen bestehende Regeln, die wir Revolte nennen, ist ihrem Wesen nach individualistisch, auch wenn viele Mitglieder einer Gruppe daran teilnehmen. Sie bedeutet persönliche Ansprüche des einzelnen auf gewisse Werte, die ihm das traditionelle System nicht gewährt. Eine revolutionäre Tendenz kann ebenfalls solche persönlichen Ansprüche beinhalten und insoweit eine Revolte sein; ihr charakteristisches Merkmal ist es jedoch, daß sie neue Werte für eine ganze Gruppe fordert" (id. 309). Cf. auch Niebuhr 36, 215. Ohne festere Grundlage scheint der marxistische Versuch zu sein, die Konfliktsituation, die sich infolge der Tätigkeit eines revoltierenden Individuums ergeben hat, so zu erklären, daß sie auch dadurch die soziale Gemeinschaft und Solidarität fördert (cf. Coser $114 \mathrm{f}$., 151).

${ }^{61}$ Cf. Biezais $1975,17 \mathrm{f}$.

${ }^{62}$ Cf. Jarvie $1970,81 \mathrm{f}$. 
derungen in den entsprechenden Situationen nicht revolutionär gewesen wären. Es ist auch nicht damit zu erklären, daß die offiziellen Vertreter des Kultes sie verfolgten.

Wenn man die Tätigkeit Jesu innerhalb desselben Volkes und in ähnlichen Situationen betrachtet, fällt einem ein großer Unterschied auf. Wir haben uns bereits zu den revolutionären Ansichten Jesu und deren Rolle geäußert. Die Frage lautet jetzt: Warum bildete sich um ihn eine neue Bewegung, und warum geschah das unter ähnlichen Umständen bei den anderen Propheten nicht? Dazu kann man mit dem bekannten Ausdruck ant-

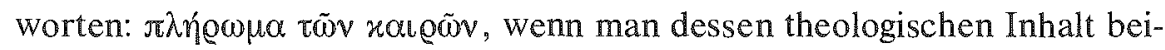
seite läßt. Dasselbe läßt sich mit einfacheren Worten ausdrücken: Es handelt sich um eine Zeiterscheinung. Die Menschen spürten es, daß irgendetwas geschehen müsse. Oder, um mit Smoliks Worten zu sprechen: „Diese Schwangerschaft mit der Revolution, deren Folgen die gewaltige revolutionäre Aktion"63 sind, ist typisch für die Zeit, in der Jesus lebte. Es ist nicht wichtig, wie wir diese Stimmung bezeichnen, ob als Erfüllung der Zeit, Schwangerschaft, revolutionäre Stimmung oder anders. Wichtig ist jedoch die Feststellung, daß es sich um eine sehr bedeutsame und notwendige Komponente handelt, damit sich um eine revolutionäre Person eine neue Bewegung bilden kann. Der Soziologe Mühlmann sagt von dieser Erscheinung, indem er sie auf eine prinzipielle Ebene stellt: „Wenn wir von 'sozialen' Bewegungen sprechen, haben wir ein psychisches, und zwar kollektiv-psychisches In-Bewegung-Kommen mit im Horizont. Der Begriff der (sozialen) Bewegung ist also psychologisch erfüllt. Ein bisher stabiler und ruhiger Zustand ist aus dem Gleichgewicht gekommen [ . . ].

Wir sagten, der Begriff der Bewegung sei psychologisch erfüllt. Und zwar meinen wir: tiefenpsychologisch mit erfüllt. Je mehr eine Bewegung 'Bewegung' ist, um so stärker ist sie bestimmt durch die psychische Tiefenschicht; und je mehr in ihr - in ihrem Verlauf - das Bewegungselement erlahmt, 'Ernüchterung' eintritt, um so mehr gelangt die Rationalschicht zur Herrschaft.

Die innere, tiefenpsychologisch erfüllte Thematik der Bewegung bezeichnen wir als Mythos. Jede echte Bewegung hat einen mehr oder weniger expressiven, stimmungsgeladenen Mythos. Wir nehmen dabei den Begriff des Mythos sowohl im religionshistorisch-ethnographischen als auch im

${ }^{63}$ Smolik 565. 
politischen Sinne." ${ }^{64}$ Mir scheint, daß er die Bedeutung der erwähnten zweiten Komponente für den Enstehungsprozeß solcher Bewegungen richtig erkannt hat. Es handelt sich um eine tiefere Schicht des psychischen Bewußtseins, die die Verantwortlichkeit einer Gruppe bestimmt. Sie schafft die Situation, in der das revolutionäre Individuum und die für die Revolution reif gewordene Gesellschaft zusammenarbeiten, sie erzeugt die besondere dynamische Situation, aus der sich die neue Bewegung entwickelt ${ }^{65}$. Daher können wir als Folgerung feststellen, daß die dynamischen Bewegungen von einzelnen Personen hervorgerufen werden und daß ihre Bildung von einer gewissen sozialen Situation mitbestimmt wird. Sie sind das Resultat der Einwirkung dieser beiden Komponenten.

6.3. Die vorhin gemachten Ausführungen über die bestimmenden Faktoren dynamischer Bewegungen helfen auch, die Prozesse besser zu verstehen, die im Verlauf der Institutionalisierung sichtbar werden. Revolutionäre Bewegungen sind ein Protest gegen die bestehende Ordnung des religiösen Lebens bzw. gegen das Wertsystem. Aber es handelt sich nicht nur um einen Protest um des Protestes willen in einer negativen Auslegung. Ein solcher Protest ist nicht als Destruktion zu verstehen. Er ist in seinen Intentionen positiv. Seine Absicht ist es, eine neue Lebensordnung zu schaffen, in unserem Fall: das traditionelle religiöse Leben durch ein anderes zu ersetzen, das nach anderen Wertmaßstäben organisiert ist. Wenn das nicht so wäre, würden die dynamischen revolutionären $B$ ewegungen zu einem nihilistischen Anarchismus führen. Das ist zwar theoretisch denkbar, hat aber mit den von uns behandelten religiösen Bewegungen nichts zu tun.

Es ist selbstverständlich, da $\beta$ es sich bei den dynamischen Bewegungen um empirische soziale Erscheinungen handelt, die nur als solche zu betrachten sind. Daher ist es auch eine rein hypothetische Annahme, daß sie in ihrem Entstehungs- und Entwicklungsgang, in großen Zügen gesehen, zu zweierlei Resultaten führen können. In einem Fall werden diese Be-

${ }^{64}$ Mühlmann 1964, 262f. Ähnlich auch id. 1975, 50.

${ }^{65}$ Hier genügen Hinweise auf einige typische Beispiele außerhalb des Christentums über die Zusammenarbeit des Individuums und der in eine dynamische Bewegung geratenen Gesellschaft in einer bestimmten Situation. So ist das zu erkennen im Falle der von Hung Hsiuch'üan geleiteten t'ai-p'ing-Bewegung (solche Beschreibungen bei Oehler, Michael, Jen Yu-wen u. a.), oder auch von Kimbangu und seinen Anhängern (Andersson), Shembe und seiner Nazaretha-Kirche (Sundkler); über weitere solche Bewegungen Lanczkowski 1971 , $56 \mathrm{ff}$.; 1974, $10 \mathrm{ff}$. 
wegungen mit Hilfe der herrschenden statischen Gesellschaft überwunden und verschwinden allmählich. Die Gesellschaft setzt dann im allgemeinen ihr Leben in Übereinstimmung mit den früheren Normen und Traditionen fort. In dieses Bild fügen sich die in der Religionsgeschichte so gut bekannten verschiedenen Häretikerbewegungen ein. Im anderen Falle sind die dynamischen Bewegungen so stark, daß die statische Gesellschaft sie nicht zu überwinden und zum früheren Zustand zurückzukehren vermag. In diesem Fall wird ihre Struktur wesentlich verändert, so daß eine neue Gesellschaft entsteht. Doch kann es hier mehrere Variationen geben: neben der wesentlichen Veränderung der alten Gesellschaft unter dem Einfluß der dynamischen Bewegungen können sich von der alten Gesellschaft abgespaltene, neue, d. h. Dissidentengruppen bilden. Diese sind in der christlichen Kirche als Sekten bekannt. Und hier ist die Frage zu stellen: Was ist in diesem Fall mit dem dynamischen Prozeß selbst geschehen? Die Antwort ist im Rahmen unseres Symposiums wichtig.

Der revolutionäre Prozeß ist immer eine sehr intensive Erscheinung. Er spielt sich mit geballter Kraft ab. Nur in einem besonderen psychischen Zustand, den ich als Zustand der Hochspannung bezeichnen möchte, ist es möglich, die Opfer zu bringen, die die revolutionären Bewegungen manchmal von den einzelnen Teilnehmern fordern. Doch kann man nicht dauernd in einer solchen Hochspannung leben. Der dynamische Prozeß ist bereits in seinem Anfangsstadium so vordisponiert, daß er nach Erreichen eines gewissen Zustandes haltmacht. Das birgt die Forderung nach einem neuen, ausgeglichenen Zustand in sich. So wird der durch den dynamischen Prozeß hervorgerufene labile Zustand überwunden. Es bedeutet, daß jede dynamische Bewegung sich schließlich stabilisiert. Diese Stabilisierung ist nichts anderes als eine Institutionalisierung. Die dynamische Bewegung geht in eine neue Institution über.

Diese neue Institution hat ihre typischen Merkmale. Wir erwähnen hier nur einige. Die Forderungen, die zu Beginn des Prozesses ausgesprochen wurden, werden in der neuen Institution niemals vollständig realisiert. Sie stabilisieren sich auf einem niedrigeren Niveau ${ }^{66}$. Das zweite Moment, das

\footnotetext{
${ }^{66}$ Mühlmann 1975, 50; „Aus religiösen Bewegungen werden Sekten oder Kirchen, aus sozialen Bewegungen Parteien, aus künstlerischen Akademien oder 'Sezessionen' usw. Bei allem kulturell Neuen ist das definitiv Neue, das schließlich zustane kommt, eine Stabilisierung oberhalb des Nullpunktes, mehr oder weniger hoch; nicht zu niedrig, sonst wäre ja der alte Zustand konserviert, nicht zu hoch, sonst könnte er nicht stabil bleiben."
} 
hier genannt werden soll, bezieht sich auf die sogenannten spontanen Äußerungen des Geistes im Zustand der Exstase wie Visionen, Glossolalien u. a. Diese wurden stets als typische Anzeichen einer dynamischen religiösen Bewegung angesehen. Im Prozeß der Institutionalisierung verschwinden sie zwar nicht völlig, doch unterliegen sie einer sehr bezeichnenden Umwertung ihrer Rolle. Diese Äußerungen des ,Geistes“ werden zum Bestandteil des Kultes. Sie werden einem streng geordneten Ritus unterworfen. Die Ritualisierung dieser Geistesäußerungen ist eines der typischen Zeichen für den Vorgang der Bildung einer Institution. Das, was zu Beginn der Bewegung als das wichtigste angesehen wurde, wird umwertet, ja, selbst mit einem gewissen Mißtrauen betrachtet. Daher ist die Regulierung solcher psychischer Äußerungen im Kult verständlich. Die dritte Tendenz bezieht sich auf die „Empfangung“ des Geistes. In messianischen und chiliastischen Bewegungen motivierten deren Führer und Anhänger ihre Tätigkeit in der Anfangszeit mit ihrer Sendung, die sie durch göttliche Inspiration und Offenbarung erfahren haben wollten. Doch im Verlauf der Institutionalisierung der Bewegungen treten andere Forderungen an ihre Stelle. Nicht alle Geister, die tätig werden und auf die man sich berufen kann, sind gut. Man muß sie überprüfen. Eine solche Prüfung erfordert stabile Richtlinien. Wegen dieser Forderung entwickeln sich dogmatische Lehrsätze und moralische Verhaltensregeln. Die einen wie die anderen sind in der Anfangszeit der Bewegung überflüssig, denn der Enthusiasmus des Geistes ist mit kategorischen Normen nicht zu unterdrücken. Auch im Urchristentum verschwanden sehr bald die enthusiastisch-pneumatischen und eschatologischen Züge zugunsten der entstehenden institutionalisierten Kirche. Das hat Harnack ganz richtig gesehen: ,,Man nahm ihnen [den Menschen] die Religion, die sie verstanden und gab ihnen dafür einen Glauben, den sie nicht verstehen konnten, resp. der alte Glaube und die alten Hoffnungen verblaßten von selber, und an ihre Stelle trat die Autorität eines geheimnisvollen Glaubens. “67

7. Werfen wir einen Blick zurück auf die hier behandelten Ansichten und geäußerten Standpunkte über die dynamischen und sich institutionalisierenden Prozesse, deren Stellung und Rolle im religiösen Leben, so können wir zu einigen prinzipiellen Erkenntnissen kommen. Zunächst ist es wichtig, sich darüber zu einigen, wie weit die beiden Begriffe gefaßt werden

${ }^{67}$ Harnack 620. 
sollen. Wir haben hier wiederholt darauf hingewiesen, daß der Mensch seinem Wesen nach dynamisch ist. Das bezieht sich ebenso auf jede soziale Erscheinung. Es ist eines der grundsätzlichen Kennzeichen des Bestehens einer jeglichen Gruppe. Die sozialen Gruppen bestehen nur durch einen ständigen Umwandlungsprozeß. So wird verständlich, daß man heutzutage, wo verschiedene religiöse Gruppen, einschließlich der Kirche, eine große Aktivität aufweisen, von Dynamik zu sprechen pflegt. Doch bezieht sich das nur auf die aktiven Prozesse. Damit ist nur der Grundzug einer jeden sozialen Erscheinung, wie sie hier erwähnt wurde, gemeint, aber nichts Spezifisches. So versteht auch der Funktionalismus die Veränderungen in der Gesellschaft, wie wir vorhin gesehen haben. Mit anderen Worten, es handelt sich um den jeder Gruppe endogenen, ununterbrochenen Entwicklungsprozeß. So verstanden, sagt der Dynamismus nichts über die grundlegenden und radikalen Veränderungen der religiösen Struktur oder, wie wir vorhin sagten, über deren revolutionären Charakter aus. Künftig müßte man sich daher davor hüten, wenn man von dynamischen Veränderungen spricht, den Begriff in diesem Sinne zu gebrauchen. So weit gefaßt, sagt sein Inhalt tatsächlich über die spezifischen dynamischen Erscheinungen nichts aus. Er wäre nur in bezug auf solche Bewegungen zu benutzen, die eine klare Tendenz aufweisen, die bestehenden Strukturen wesensmäßig zu verändern.

Das gleiche ist auch über den Gebrauch des Begriffs Institution zu sagen. Wie wir gesehen haben, ist jede dynamische religiöse Bewegung nicht nur mit dieser verbunden, sondern sie erwächst auch aus einer stabilen Einheit. Unter den Forschern ist die Tendenz zu beobachten, anzunehmen, $\mathrm{da} ß$ jede dynamische Bewegung eo ipso einer Tradition angehört. Mit anderen Worten: die institutionelle Gruppe ist es, die schon vorher den Charakter der dynamischen Prozesse bestimmt. Es ist nicht zu leugnen, daß dieser Standpunkt seine Grundlage hat. Keine dynamische Bewegung ist anders $\mathrm{zu}$ verstehen als aus dem richtigen historischen und sozialen $\mathrm{Ge}-$ samtzusammenhang heraus. Aber das bedeutet noch bei weitem nicht, daß dieser Zusammenhang den Inhalt der dynamischen Bewegung selbst bestimmen würde. Es hat sich gezeigt, daß unter den gleichen gegebenen historischen Verhältnissen dynamische Bewegungen den Charakter von religiösen, politischen oder ökonomischen Bewegungen annehmen können. Das bedeutet, daß ein und dieselbe traditionelle statische Grundlage die Bildung verschiedener dynamischer Bewegungen zuläßt. Daher ist auch, 
wenn man von Institutionalisierung spricht, der Begriff der Institution abzugrenzen. Er ist in der Weise einzuschränken, daß er wohl als Grundlage anzusehen ist, aus der die dynamischen Bewegungen erwachsen, der aber dem Wesen nach deren Inhalt nicht bestimmt.

Nur wenn man beide Begriffe auf ihren spezifischen Inhalt beschränkt, treten deren eigentliche Beziehungen hervor. Einerseits stehen sie stets miteinander in Relation. Oder, noch deutlicher, der eine Begriff ist vom anderen abhängig. Andererseits aber ist diese Relation weder kausal noch final. Sie ist dialektisch. Es ist die Aufgabe der einzelnen Beiträge dieses Symposiums zu zeigen, welche Faktoren und Momente in jedem einzelnen konkreten Fall die dialektischen Beziehungen dieser Begriffe bestimmen.

\section{Literaturverzeichnis}

AJS American journal of sociology.

ASR American sociological review.

JSR Journal for the scientific study of religion.

ST Soziologische Texte. Darmstadt.

UT Urban-Taschenbücher. Stuttgart.

Agassi, J., 1960, Methodological individualism. The British journal of sociology 11.

Behrendt, R., 1973, Der Mensch im Licht der Soziologie. UT 60.

Berger, P., 1963, Charisma and religious innovation: the social location of Israelite prophecy. $A S R 28$.

Berger, P., Luckmann, T., 1963 a, Sociology of religion and sociology of knowledge. Sociology and social research 47.

Biezais, H., 1939, Dzīivibas un milestïbas sinteze kristīgajā humanitātē. Summary: The Christian valuation of the ethical principles of biocentrical philosophers. Diss. Rĩgā.

- 1963, Kristiānisma vēsturiskã vide [Das geschichtliche Umgebung des Christentums]. Ieskatītais un atzìtais. Red. H. Biezais. Stokholmā.

- 1965, Den ryska kommunismens syn på kristendomen. Kristendomslärarnas förenings årsbok 9. Uppsala.

- 1965 a, Kultūras religiskās saknes [Die religiösen Wurzeln der Kultur]. Tèvzemes brīvībai un kultürai. Indianopolē.

- 1969, Tendenser i nutida messianska rörelser. Religion och Bibel 28. Lund.

- 1971, Nathan Söderblom och den religionshistoriska forskningens genombrott. Arsskrift utg. av Åbo Akademi 1969-1970. Åbo.

- 1971 a, Nathan Söderblom och den religionshistoriska forskningens genombrott. Religion och kultur 42.

- 1975, Zur Problematik der neuen Religionen. New religions. Ed. H. Biezais. Scripta Instituti Donneriani Aboensis 7. Uppsala.

Bredermeier, H., 1955, The methodology of functionalism. ASR 20.

Buckley, W., 1958, Social stratification and the functional theory of social differentiation. ASR 23.

- 1963, On equitable inequality. ASR 28. 
Bultmann, R., 1954, Das Urchristentum im Rahmen der antiken Religionen. Zürich.

Carlsson, G., 1958, Social mobility and class structure. Lund.

- 1962, Reflections on functionalism. Acta Sociologica 5.

Carmichael, J., 1962, The death of Jesus. New York.

Civilisation, 1930, Civilisation: le mot et l'idée. Centre international de synthèse. Première semaine internationale de synthèse 2. Paris.

Coser, L., 1956, The functions of social conflict. Glencoe.

Dahrendorf, R., 1961, Über den Ursprung der Ungleichheit unter den Menschen. Recht und Staat in Geschichte und Gegenwart 232. Tübingen.

Davis, K., 1942, A conceptual analysis of stratification. ASR 7.

Davis, K., Moore, W., 1945, Some principles of stratification. ASR 10.

Desroche, H., 1968, Sociologies religieuses. Paris.

- 1973, Sociologie de l'espérance. Paris.

Dillenberger, J., 1966, Paul Tillich: theologian of culture. Paul Tillich, Retrospect and future. Intr. by T. Kantonen. Nashville and New York.

Eickelpasch, R., 1973, Mythos und Sozialstruktur. Studien zur Sozialwissenschaft 7. Düsseldorf.

Eisler, R., 1930, Iesous basileus ou basileusas 2. Religionswissenschaftliche Bibliothek 9. Heidelberg.

Febvre, L., 1930, Civilisation. Évolution d'un mot et d'un groupe d'idées. Civilisation.

Functionalism, 1965, Functionalism in the social sciences. Ed. by D. Martindale. The American academy of political and social sciences. Monograph 5. Philadelphia.

Geiger, T., 1967, Die soziale Schichtung des deutschen Volkes. Stuttgart.

Goldschmidt, W., 1966, Comparative functionalism. Berkeley and Los Angeles.

Greschat, H.-J., 1968, Legend? Fraud? Reality? Alice Lenshina's prophetic experience. Africana Marburgensia 1.

Harnack, A., 1909, Lehrbuch der Dogmengeschichte 1. Heidelberg.

Hauser, R., 1967, Utopie und Hoffnung. Säkularisation.

Hayek, F., 1962, The counter-revolution of science. Glencoe.

Heinichen, O., 1924, Driesch's Philosophie. Leipzig.

Hempel, C., 1959, The logic of functional analysis. Symposium.

Holmström, F., 1937, Uppenbarelsereligion och mystik. En undersökning av Nathan Söderbloms teologi. Stockholm.

Holsten, W., 1966, Zum Verhältnis von Religionswissenschaft und Theologie. Festschrift Walter Batke. Weimar,

Jarvie, I., 1965, Limits to functionalism and alternatives to it in anthropology. Functionalism.

- 1970, The revolution in anthropology. London.

Jordan, P., 1967, Religion und Ideologie als geschichtliche Wirkmächte. Säkularisation.

Käsemann, E., 1962, Exegetische Versuche und Besinnungen 1. Göttingen.

Kamlah, W., 1969, Utopie, Eschatologie, Geschichtsteleologie. B $\cdot$ I-Hochschultaschenbücher 461. Mannheim.

Körber, S., 1976, Bedingtheit und Distanzbemühen. Der Religionswandel unserer Zeit im Spiegel der Religionswissenschaft. Darmstadt.

Kolb, W., 1953, Values, positivism, and the functional theory of religion: the growth of a moral dilemma. Social forces 31 (=Yinger 1967).

Kroeber, A., Kluckhohn, C., 1963, Culture. Vintage books V-226. New York.

Lanczkowski, G., 1971, Begegnung und Wandel der Religionen. Düsseldort:

- 1974, Die neuen Religionen. Bücher des Wissens 6237. Frankfurt a. M. 
Lanternari, V., 1965s, Syncrétismes, messianismes, néo-traditionalismes. Archives de sociologie des religions $10 \mathrm{~s}$.

- 1966, Religiöse Freiheits- und Heilsbewegungen unterdrückter Völker. ST 33.

Linton, R., 1957, The tree of culture. New York.

Luckmann, T., 1972, The invisible religion. New York and London.

Malinowski, B., 1931, Culture. Encyclopaedia of the social sciences 4. New York.

- 1936, The foundations of faith and morals. Riddell memorial lectures 7 (1934-5). London.

- 1948, Magic, science and religion, and other essays. New York.

Martindale, D., 1965, Limits of and alternatives to functionalism in sociology. Functionalism.

Mensching, G., 1959, Die Religion. Stuttgart.

Moore, W., 1963, But some are more equal than others. ASR 28.

Mühlmann, W., 1964, Chiliasmus und Nativismus. Studien zur Soziologie der Revolution 1. Berlin.

- 1975, Zwischen Exzess und Ordnung. Die Gehäuse des Menschen. Hrsg. G.-K. Kaltenbrunner. Herderbücherei Initiative 9. Freiburg.

Niebuhr, R., 1932, Moral man and immoral society. New York.

O'Dea, T., 1961, Five dilemmas in the institutalization of religion. JSR 1.

- 1964, Die fünf Dilemmas der Institutionalisierung der Religion. Religionsoziologie. Hrsg. F. Fürstenberg. ST 19. Neuwied (=Social compass 7 (1960)).

- 1970, Sociology and the study of religion. New York and London.

Ogburn, W., 1936, Stationary and changing societies. AJS 42.

Parsons, T., 1940, An analytical approach to the theory of social stratification. AJS 45.

- 1957, A revised analytical approach to the theory of social stratification. Bendix, R., Lipset, S., Class, status and power. Glencoe.

Radcliffe-Brown, A., 1948, The Andaman islanders. Glencoe.

- 1969, Structure and function in primitive society. London.

Religion, 1964, Religion and social conflict. Ed. by R. Lee, M. Marty. New York.

Rosenkranz, G., 1967, Der christliche Glaube angesichts der Weltreligionen. Bern und München.

Säkularisation, 1967, Säkularisation und Utopie. [Festschrift für] E. Forsthoff. Stuttgart.

Savramis, D., 1968, Religionssoziologie. München.

Scharf, Betty, 1970, The sociological study of religion. London.

Schlick, M., 1971, Über den Begriff der Ganzheit. Soziologie.

Smith, A., 1973, The concept of social change. London and Boston.

Smolik, J., 1969, Das theologische Problem der Revolution. Concilium 5.

Söderblom, N., 1907, Studiet av religionen. Stockholm.

Sorokin, P., 1964, Social and cultural mobility. New York.

Soziologie, 1971, Soziologie. Logik der Sozialwissenschaften. Hrsg. E. Topitsch. Neue wissenschaftliche Bibliothek 6. Köln.

Spencer, R., 1965, The nature and value of functionalism in anthropology. Functionalism.

Stähli, M., 1976, Reich Gottes und Revolution. Theologische Forschung 57. Hamburg.

Steinbacher, F., 1971, Die Gesellschaft. UB 812.

Steinbauer, F., 1970, Die Cargo-Kulte im Melanesien 1. Nürnberg.

Steinbeck, Brigitte, 1964, Einige Aspekte des Funktionsbegriffes in der positiven Soziologie und in der kritischen Theorie der Gesellschaft. Soziale Welt 15.

Stinchcombe, A., 1968, Constructing social theories. New York.

Symposium, 1959, Symposium on sociological theory. Ed. by Llewellyn Gross. Evanston. 
Talmon, Yonina, 1962, Pursuit of the millennium: the relation between religious and social change. Archives européennes de sociologie 3.

Thomas, W., 1965, Person und Sozialverhalten. ST 26. (auch Ścial behavior and personality, ed. by E. Volkart. 1951).

Tillich, P., 1967, Die religiöse Substanz der Kultur. Gesammelte Werke 9. Stuttgart (=Philosophische Vorträge veröffentlicht von der Kant-Gesellschaft 24 (1920)).

Tonnelat, E., 1930, Kultur. Histoire du mot, évolution du sens. Civilisation.

Topitsch, E., 1972, Vom Ursprung und Ende der Metaphysik. Wissenschaftliche Reihe 4105. Nördlingen.

- 1973, Gottwerdung und Revolution. Uni-Taschenbücher 288. Pullach.

Tumin, M., 1953, Some principles of stratification: a critical analysis. ASR 18.

Vicedom, G., 1957, Världsreligionernas angrepp på kristendomen. Stockholm.

Volz, P., 1949, Prophetengestalten des Alten Testaments. Stuttgart.

Wiehn, E., 1968, Theorien der sozialen Schichiung. München.

Wiehn, E., Mayer, K., 1975, Soziale Schichtung und Mobilität. Beck'sche schwarze Reihe 132. München.

Windelband, W., 1957, Lehrbuch der Geschichte der Philosophie. Tübingen.

Worsley, P., 1968, The trumpet shall sound. New York.

Yinger, J., 1961, Comment. JSR 1.

- 1963, Sociology looks at religion. New York and London.

- 1967, Religion, society and the individual. New York.

- 1970, The scientific study of religion. London. 